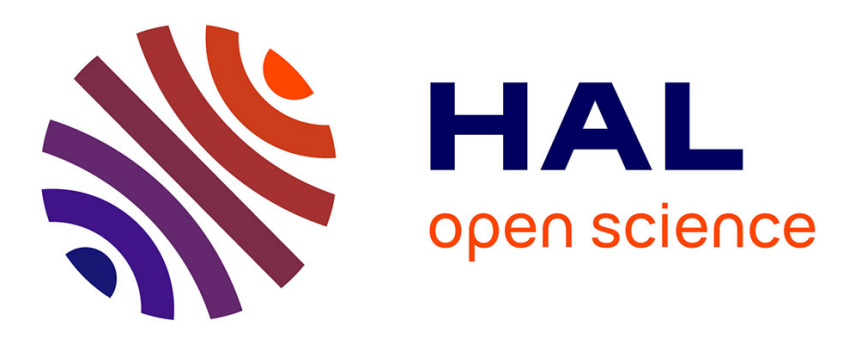

\title{
A Lightweight Epistemic Logic and its Application to Planning
}

Martin Cooper, Andreas Herzig, Faustine Maffre, Frédéric Maris, Elise

Perrotin, Régnier Pierre

\section{- To cite this version:}

Martin Cooper, Andreas Herzig, Faustine Maffre, Frédéric Maris, Elise Perrotin, et al.. A Lightweight Epistemic Logic and its Application to Planning. Artificial Intelligence, 2021, Special issue: SI: Ethics for Autonomous Systems, 298, pp.103437. 10.1016/j.artint.2020.103437 . hal-03147798

\section{HAL Id: hal-03147798 \\ https://hal.science/hal-03147798}

Submitted on 15 Feb 2022

HAL is a multi-disciplinary open access archive for the deposit and dissemination of scientific research documents, whether they are published or not. The documents may come from teaching and research institutions in France or abroad, or from public or private research centers.
L'archive ouverte pluridisciplinaire HAL, est destinée au dépôt et à la diffusion de documents scientifiques de niveau recherche, publiés ou non, émanant des établissements d'enseignement et de recherche français ou étrangers, des laboratoires publics ou privés. 


\title{
A Lightweight Epistemic Logic and its Application to Planning
}

\author{
Martin C. Cooper ${ }^{\mathrm{a}}$, Andreas Herziga ${ }^{\mathrm{a}}$ Faustine Maffre ${ }^{\mathrm{a}}$, Frédéric Maris ${ }^{\mathrm{a}}$, \\ Elise Perrotin ${ }^{a}$, Pierre Régnier ${ }^{a}$ \\ ${ }^{a}$ IRIT, CNRS, Univ. Paul Sabatier, 118 Route de Narbonne, F-31062 Toulouse Cedex 9, France
}

\begin{abstract}
We study multiagent epistemic planning with a simple epistemic logic whose language is a restriction of that of standard epistemic logic. Its formulas are boolean combinations of observability atoms: sequences of 'knowing whether' operators followed by propositional variables. This compares favourably with other restricted languages where formulas are boolean combinations of epistemic literals: sequences of 'knowing that' epistemic operators and negations followed by propositional variables; or in other terms: epistemic formulas without conjunctions or disjunctions. The reason is that our language enables a richer theory of mind: we can express statements such as "I don't know whether $p$, but I know that you know whether $p$ " which are important in communication and more generally in interaction and which cannot be expressed with epistemic literals. Going beyond previous work, we also introduce a 'common knowledge whether' operator. We show that satisfiability is nevertheless NP-complete. We then define simple epistemic planning tasks as generalisations of classical planning tasks: action descriptions have sets of observability atoms as addand delete-lists, initial states are sets of observability atoms, and goals are boolean combinations of observability atoms. We show that simple epistemic planning tasks can be polynomially translated into classical planning tasks. It follows that checking solvability of simple epistemic planning
\end{abstract}

\footnotetext{
*Corresponding author: Andreas Herzig, Andreas.Herzig@irit.fr
} 
tasks is PSPACE-complete. We present some application examples such as the gossip problem and some experimental results and clarify the relationship with Dynamic Epistemic Logic-based planning.

Keywords: Epistemic planning; multiagent planning; epistemic logic; gossip problem

\section{Introduction}

Suppose there are $n$ agents, each of which knows some secret: a piece of information that is not known to the others. They communicate by phone calls, and whenever one person calls another they tell each other all they

5 know at that time. How many calls are required before each item of gossip is known to everyone? This is known as the gossip problem. It is of great interest in the conception of communication networks and in parallel and distributed computing, but there are other less obvious applications like the management of data on storage devices [1], or the computation of the syntenic distance between two genomes (minimum number of fusions, fissions, and translocations required to transform one into the other) [2]. Several variants have been studied in the literature, and a survey of these alternatives and the associated results has been published [3]. The gossip problem can be viewed as a multiagent epistemic planning task where the goal is shared knowledge: everybody knows all secrets. It is a purely epistemic planning task because it is only the agents' knowledge that evolves, while the facts of the world remain unchanged. There are numerous variations of such a planning task; see e.g. [4] for an overview. The goal may in particular be to achieve higher-order shared knowledge: everybody knows that everybody knows all secrets, and so on. We believe that generalised gossiping is a paradigmatic epistemic planning task, much in the same way as the blocksworld is a paradigmatic classical planning task: (1) it is multiagent; (2) it is 'truly epistemic', in the sense that it involves higher-order knowledge; (3) it is as simple as it could be, in the sense that there are no 
ontic actions; (4) it has many variations.

The aim of the present paper is to introduce a simple logical approach within which we can account for centralised epistemic planning tasks such as the above. We could have chosen Dynamic Epistemic Logic (DEL) for this purpose, as done by [5]: its event models allow us to model telephone calls as private announcements. However, we take inspiration from a simpler framework: the Dynamic Epistemic Logic of Propositional Assignment and Observation (DEL-PAO), introduced in [6] and further developed in [7, 8, 9]. It is based on observability atoms, that are recursively defined as either propositional variables or atomic pieces of information about whether or not an agent sees the truth value of a observability atom. DEL-PAO was applied to epistemic planning in [10]. We here simplify this account by disregarding the dynamic aspects and concentrating on the epistemic logic underlying DEL-PAO. That logic is consequently called the Epistemic Logic of Observation, abbreviated as EL-O. The present paper improves over [10] by simpli-

fying the semantics (models are no longer infinite), by integrating a form of common knowledge into planning, and by a complexity analysis: satisfiability is NP-complete. Our EL-O-based definition of simple epistemic planning tasks parallels that of classical planning tasks, with actions, initial state, and goal described by means of observability atoms. This allows us to polynomially translate the former into the latter. It follows that deciding the solvability of a simple epistemic planning task is PSPACE-complete (just as DEL-PAO satisfiability, validity and model checking are).

The paper is organised as follows. In Section 2 we provide a detailed motivation of our approach and briefly overview related work. In Section 3 we introduce EL-O and in Section 4 we establish that it is a fragment of the standard epistemic logic S5 with an NP-complete satisfiability problem. In Section 5 we give a formal definition of action descriptions and simple epistemic planning tasks within our framework and polynomially translate them into classical planning tasks, from which PSPACE membership of deciding plan existence follows. In Section 6 we apply our framework 
to examples, in particular the gossip problem, and in Section 7 we report on some experiments that were done via an encoding of simple epistemic planning tasks into PDDL. Section 8 discusses related work, where we argue in particular that our action descriptions are a more appropriate way to describe the behaviour of actions than with DEL event models. Section 9 concludes.

\section{Background and motivation}

Reasoning in epistemic logic as introduced by Hintikka [11] and popularised in AI by Halpern and colleagues [12] is strictly more complex than in classical logic: the satisfiability problem is at least in PSPACE, and is EXPTIME-complete if common knowledge is involved [13]. The complexity gap widens for planning tasks: while the solvability problem is PSPACEcomplete for classical planning [14], it is undecidable for planning with DEL-based event models [15]. The latter provide rather expressive models for the agents' perception of actions. They parallel the standard epistemic logic modelling of uncertainty by indistinguishability relations between possible states: uncertainty in the perception of actions is modelled by indistinguishability relations between possible events. As explored by several authors in a series of papers, undecidability of DEL planning is already the case under very weak hypotheses about these event models [16, 17, 18, 19]. Basically, DEL planning tasks are only decidable when the event model is a singleton, i.e., when all actions are public. However, actions in the gossip problem are not public, nor are they in many real world applications.

We propose to base epistemic planning on an epistemic logic that is simpler than Hintikka's: epistemic information is restricted to the atomic formulas of the language. We are not the first to do this: several previous approaches have proposed languages where the scope of the epistemic operator $K_{i}$ is restricted to literals, or literals that are preceded by a sequence of 
epistemic operators [20, 21, 22, 23], possibly with negations [24, 25]. Such restrictions however exclude formulas such as $K_{i}\left(K_{j} p \vee K_{j} \neg p\right)$ expressing that agent $i$ knows that agent $j$ knows whether the propositional variable $p$ is true. This is a major drawback because such formulas are fundamental in communication and more generally in any form of interaction: a situation where agent $i$ does not know whether $p$ is the case or not $\left(\neg K_{i} p \wedge \neg K_{i} \neg p\right)$ but knows that $j$ knows $\left(K_{i}\left(K_{j} p \vee K_{j} \neg p\right)\right)$ may lead agent $i$ to ask $j$ about $p$.

We here make a similar restriction, but move from the primitive 'knowingthat' operator $K_{i}$ to the less standard 'knowing-whether' or 'knowing-if' operator $\mathrm{Kif}_{i}$. In the unrestricted language these two operators are interdefinable: we have $K i f_{i} \varphi \leftrightarrow K_{i} \varphi \vee K_{i} \neg \varphi$ and $K_{i} \varphi \leftrightarrow \varphi \wedge K_{i f} \varphi$, for arbitrary formulas $\varphi$ [26]. However, the situation changes when we restrict the language to sequences of 'knowing-whether' operators followed by propositional variables. On the one hand, any sequence of 'knowing-that' operators followed by a literal can be expressed by a sequence of 'knowing-whether' operators:

- $K_{i} p$ is equivalent to $p \wedge K i f_{i} p$

- $K_{i} \neg p$ is equivalent to $\neg p \wedge K i f_{i} p$;

- $K_{i} K_{j} p$ is equivalent to $p \wedge K i f_{i} p \wedge K i f_{j} p \wedge K i f_{i} K i f_{j} p$;

- and so on.

On the other hand and as we will prove in Section 8.2 , from $n \geq 2$ on sequences $K i f_{i_{1}} \ldots K i f_{i_{n}} p$ of 'knowing-whether' operators followed by propositional variables cannot be expressed by means of boolean combinations of 'knowing-that' operators followed by literals. In particular, no such boolean combination is equivalent to $\mathrm{Ki}_{i} K i f_{j} p$. Such increased expressivity makes a difference. For example, consider the above situation where $i$ does not know whether $p$ but knows whom to ask, as expressed by the formula $\neg K_{i} p \wedge \neg K_{i} \neg p \wedge K_{i}\left(K_{j} p \vee K_{j} \neg p\right)$ : this can be expressed in our language by $\neg K i f_{i} p \wedge K i f_{j} p \wedge K i f_{i} K i f_{j} p$. 
Instead of $K i f_{i}$ we prefer to write $S_{i}$, and we read $S_{i} p$ as "agent $i$ sees whether $p$ is true or not". This highlights that when we define $K_{i} p$ as $p \wedge S_{i} p$,

implying the infinite set of propositions of the form $S_{i_{1}} \ldots S_{i_{n}} p$ for all $i_{1}, \ldots, i_{n}$ and all $n \geq 1$. Suppose $p$ stands for 'the door is open'. We can imagine that $J S p$ is true when all agents are present in the same room and not only 
observe the door, but also mutually observe each other. In this concrete example, if an agent leaves the room and closes the door behind her, $J S p$ becomes false because the agents no longer mutually observe each other (even if the agent who has left can see the closed door from the outside). In order to guarantee that joint observability of $p$ implies individual observability of $p$, we require in our semantics that states containing $J S p$ also contain every $S_{i} p$, and more generally every $p$ preceded by any sequence of $S_{i}$ and $J S$. Defining $C K p$ as $p \wedge J S p$, we obtain a common knowledge operator that satisfies all the standard properties except the induction axiom $C K\left(p \rightarrow \bigwedge_{i \in \mathbb{A}} K_{i} p\right) \rightarrow(p \rightarrow C K p)$. We will discuss this issue in Section 4 (Remark 4).

It is generally assumed in the AI literature that a reasonable epistemic logic should satisfy introspection: the formulas $K_{i} \varphi \rightarrow K_{i} K_{i} \varphi$ and $\neg K_{i} \varphi \rightarrow$ $K_{i} \neg K_{i} \varphi$ should both be valid 1 In terms of observability this means that states should contain $S_{i} S_{i} p$ for every agent $i$ and propositional variable $p$, and more generally every $S_{i} S_{i} S_{j} p, S_{j} S_{i} S_{i} p$, etc. Similarly, they should also contain JS JS $p$. Moreover, whenever a state contains some JS $p$ then it should also contain $S_{i} p$, for every agent $i$. Clearly, these closure conditions make states infinite. We are going to avoid this by integrating closure into the truth condition for observability atoms.

All we have said up to now was about how to describe the agents' static knowledge. We complete the picture by adding actions that may modify both the state of the world and the agents' vision: we simply add and delete atoms. This requires some care: we have to guarantee that the above properties of introspection and 'joint sight implies individual sight' are preserved. We do so by requiring action descriptions to be consistent: they cannot delete introspective atoms such as $S_{i} S_{i} p$, they cannot add $J S p$

\footnotetext{
${ }^{1}$ We are aware that negative introspection was criticised in the literature as being too strong [30, 31]. One may however argue with Holliday [32, footnote 4] that these criticisms do not apply to observability-based knowledge.
} 
and delete $S_{i} p$ at the same time, etc. A further issue that arises when describing epistemic actions is that the description of effects should cater for conditional effects: whether or not an action changes the knowledge of some agent $i$ depends on what $i$ observes when the action is performed. For example, when agent $i$ switches the light on then the effect of that action on agent $j$ 's knowledge depends on whether the latter is in the room or not. Another example is a call between two agents in the gossip problem where the agents share all the secrets they know: what is learned is conditional on the agents' prior knowledge. Based on such action descriptions, a simple epistemic planning task is going to be made up of:

- a vocabulary;

- a set of consistent descriptions of actions with conditional effects which add and delete observability atoms;

- an initial state which is an EL-O valuation;

- a goal formula which is an EL-O formula.

Classical planning tasks are particular cases of simple epistemic planning tasks; therefore the existence of a solution to a simple epistemic planning task is PSPACE-hard. It is actually PSPACE-complete: as we will show in Section 5.5, simple epistemic planning tasks can be polynomially reduced to classical planning tasks.

\section{EL-O: Epistemic Logic of Observation}

We introduce the Epistemic Logic of Observation, abbreviated to EL-O. Its language consists of formulas over a vocabulary of observability atoms: propositional variables prefixed by sequences of observability operators $S_{i}$ and $J S$. Models of EL-O are sets of such observability atoms. The interpretation of formulas is defined in a way such that introspection principles become valid. We show that the EL-O satisfiability problem is NP-complete. 


\subsection{Boolean languages and Classical Propositional Calculus CPC}

The language $\mathcal{L}_{\text {bool }}(\mathbb{V})$ of boolean formulas over some vocabulary $\mathbb{V}$ combines the elements of $\mathbb{V}$ by means of the boolean operators. It is defined by the grammar

$$
\mathcal{L}_{\text {bool }}(\mathbb{V}): \varphi::=p|\neg \varphi|(\varphi \wedge \varphi)
$$

where $p$ ranges over $\mathbb{V}$. The boolean operators $T, \perp, \vee, \rightarrow$, and $\leftrightarrow$ are defined

of agents. The set of observability operators is OBS $=\left\{S_{i}: i \in \mathbb{A}\right\} \cup\{J S\}$, where $S_{i}$ stands for individual observability of agent $i$ and $J S$ stands for 
joint observability of all agents. The set of all sequences of observability operators is noted OBS* and the set of all non-empty sequences is noted $\mathrm{OBS}^{+}$. We use $\sigma, \sigma^{\prime}$, etc. to denote elements of $\mathrm{OBS}^{*}$ and reserve nil for the empty sequence. The length of a sequence of observability operators is defined inductively by: $\ell($ nil $)=0 ; \ell\left(S_{i} \sigma\right)=\ell(\sigma)+1$; and $\ell(J S \sigma)=\ell(\sigma)+1$.

Observability atoms, or atoms for short, are finite sequences of observability operators followed by a propositional variable. The set

$$
\mathrm{OA}=\left\{\sigma p: \sigma \in \mathrm{OBS}^{*}, p \in \mathbb{P}\right\}
$$

is the set of all atoms. (It depends on the set of propositional variables $\mathbb{P}$ and on the set of agents $\mathbb{A}$; we however leave these arguments implicit.) We use the small Greek letters $\alpha, \alpha^{\prime}, \beta, \ldots$ to denote atoms. Here are some examples: $S_{1} p$ reads " 1 sees the value of $p$ ". Hence 1 knows whether $p$ is true or false. $J S S_{2} q$ reads "all agents jointly see whether agent 2 sees the value of $q$ ". In other words, there is joint attention in the group of all agents concerning 2's observation of $q$ : agent 2 may or may not see the value of $q$, and in both cases this is jointly observed. $S_{1} S_{2} S_{3} p$ reads "1 sees whether 2 sees whether 3 sees $p$ ". The length of an observability atom is $\ell(\sigma p)=\ell(\sigma)+1$. Therefore an atom of length 1 is nothing but a propositional variable. For example, $\ell\left(J S S_{2} p\right)=3$. As $T$ abbreviates $\neg(p \vee \neg p)$, it is of length $\ell(T)=2 \times \ell(p)+3$.

Example 1. In the initial states $s_{0}^{G_{1}}$ of the gossip problem (in which secrets may or may not be true) each agent only knows her own secret. Therefore $s_{0}^{G_{1}}=\left\{S_{i} s_{i}: i \in \mathbb{A}\right\} \cup S$ where $S$ is some subset of $\left\{s_{i}: i \in \mathbb{A}\right\}$.

As explained in the introduction, individual introspection is expressed with our 'knowing-whether' operator as $S_{i} S_{i} \alpha$. Joint introspection is expressed by $J S J S \alpha$. The latter implies $\sigma J S \alpha$ for every non-empty $\sigma$ because joint observability implies any nesting of individual observability. We therefore call an atom introspective if it contains two consecutive $S_{i}$, or a $J S$ that is preceded by a non-empty sequence of observability operators. In other words, an atom is introspective if it is of the form $\sigma S_{i} S_{i} \alpha$ for some $\sigma \in$ OBS$^{*}$, 
or of the form $\sigma J S \alpha$ for some $\sigma \in \mathrm{OBS}^{+}$. The set of all introspective atoms is $\mathrm{I}-\mathrm{OA}=\left\{\sigma S_{i} S_{i} \alpha: \sigma \in \mathrm{OBS}^{*}\right.$ and $\left.\alpha \in \mathrm{OA}\right\} \cup\left\{\sigma J S \alpha: \sigma \in \mathrm{OBS}^{+}\right.$and $\left.\alpha \in \mathrm{OA}\right\}$.

The set I-OA is infinite and is a proper subset of OA.

\subsection{Atomic consequence and introspectively closed states}

We define a relation of atomic consequence between observability atoms as follows:

$$
\alpha \Rightarrow \beta \text { iff } \alpha=\beta \text {, or } \alpha=J S \alpha^{\prime} \text { and } \beta=\sigma \alpha^{\prime} \text { for some } \sigma \in \mathrm{OBS}^{+} \text {. }
$$

If this is the case then we say that $\alpha$ is a cause of $\beta$ and that $\beta$ is a consequence of $\alpha$. For example, $J S p \Rightarrow S_{i} p$ and $J S p \Rightarrow J S S_{i} p$. The relation $\Rightarrow$ is clearly reflexive and transitive. Moreover, the set of introspective atoms is closed under atomic consequence: if $\alpha \in \mathrm{I}-\mathrm{OA}$ and $\alpha \Rightarrow \beta$ then $\beta \in \mathrm{I}-\mathrm{OA}$.

We let $\alpha^{\Rightarrow}$ denote the set of consequences of $\alpha$ and $\alpha^{\Leftarrow}$ the set of its causes:

$$
\alpha^{\Rightarrow}=\{\beta: \alpha \Rightarrow \beta\}, \quad \alpha^{\Leftarrow}=\{\beta: \beta \Rightarrow \alpha\} .
$$

Here are some examples:

$$
\begin{aligned}
(p)^{\Rightarrow} & =\{p\}, & (p)^{\models} & =\{p\}, \\
\left(S_{i} p\right)^{\Rightarrow} & =\left\{S_{i} p\right\}, & \left(S_{i} p\right)^{\models} & =\left\{S_{i} p, J S p\right\}, \\
(J S p)^{\Rightarrow} & =\left\{\sigma p: \sigma \in \mathrm{OBS}^{+}\right\}, & (J S p)^{\models} & =\{J S p\}, \\
\left(S_{i} S_{j} p\right)^{\Rightarrow} & =\left\{S_{i} S_{j} p\right\}, & \left(S_{i} S_{j} p\right)^{\models} & =\left\{S_{i} S_{j} p, J S S_{j} p, J S p\right\} .
\end{aligned}
$$

Observe that $\alpha^{\Leftarrow}$ is always finite while $\alpha^{\Rightarrow}$ is either infinite (namely when $\alpha$ starts by $J S$ ) or the singleton $\{\alpha\}$ (namely when $\alpha$ is a propositional variable or starts by some $S_{i}$ ). When $\beta \Rightarrow \alpha$ then the length of $\beta$ is less than or equal to the length of $\alpha$. Moreover, the set of causes of $\alpha$ has at most $\ell(\alpha)$ elements: $\left|\alpha^{\Leftarrow}\right| \leq \ell(\alpha)$. It follows that the sum of the lengths of all causes of $\alpha$ is at most quadratic in the length of $\alpha$ :

Proposition 1. For every $\alpha, \sum_{\beta: \beta \Rightarrow \alpha} \ell(\beta) \leq(\ell(\alpha))^{2}$. 
We generalize atomic consequence to sets of atoms $s \in 2^{\mathrm{OA}}$, alias states: $s \Rightarrow=\bigcup_{\alpha \in s} \alpha$. When $s$ contains all its atomic consequences and all introspective atoms then we say that $s$ is introspectively closed or, for short, introspective. The set of all introspective states is

$$
I \text {-STATES }=\left\{s \Rightarrow \cup \mathrm{I}-\mathrm{OA}: s \in 2^{\mathrm{OA}}\right\} .
$$

$\bigwedge_{i, j, r \in \mathbb{A}}\left(S_{j} s_{i} \wedge S_{r} S_{j} s_{i}\right)$, i.e., every agent $r$ knows that every $S_{j} s_{i}$ is true.

We recall that a state is a subset of the vocabulary OA. A way of guaranteeing introspection was proposed in [6] where formulas are interpreted exclusively in the set of introspectively closed states I-STATES. As such states are always infinite, it is not immediately clear how to define model checking (which requires finite states). One way out is to work with 'sufficiently introspective states', as done in [9, Chapter 2]. Here we work with finite models and instead interpret formulas in such a way that introspection is simulated. The EL-O truth conditions are just as those for CPC of Section 3.1. except that for atomic formulas we stipulate:

$$
s \vDash \alpha \text { iff } \alpha \in s^{\Rightarrow} \cup \mathrm{I}-\mathrm{OA} .
$$


Hence $\alpha$ is true in $s$ if and only if $\alpha$ is introspective or $\beta \Rightarrow \alpha$ for some $\beta \in s$.

The EL-O semantics coincides with the CPC semantics for the fragment $\mathcal{L}_{\text {bool }}(\mathbb{P})$ of $\mathcal{L}_{\text {bool }}(\mathrm{OA})$ :

Proposition 2. Let $\varphi \in \mathcal{L}_{\text {bool }}(\mathbb{P})$. Then $s \vDash \varphi$ iff $s \models^{\mathrm{CPC}} \varphi$.

Example 3. Let $s_{0}^{G_{1}}$ be the initial state of the gossip problem from Example 1 Then $s_{0}^{G_{1}} \vDash S_{i} s_{i} \wedge \bigwedge_{j \neq i} \neg S_{i} s_{j}$ for every $i \in \mathbb{A}$.

A formula $\varphi \in \mathcal{L}_{\text {bool }}(\mathrm{OA})$ is EL-O satisfiable iff $s \vDash \varphi$ for some $s \in 2^{\mathrm{OA}}$; it is EL-O valid iff $s \vDash \varphi$ for every $s \in 2^{\mathrm{OA}}$. Clearly, an atom $\alpha$ is EL-O valid if and only if it is introspective. Moreover, for atoms $\alpha, \beta \in \mathrm{OA}$ we have that $\alpha \rightarrow \beta$ is EL-O valid iff $\alpha \Rightarrow \beta$ or $\beta$ is introspective.

Proposition 3. Let $A, B \subseteq \mathrm{OA}$ be such that $\left(\bigwedge_{\alpha \in A} \alpha\right) \wedge\left(\bigwedge_{\beta \in B} \neg \beta\right)$ is EL-O unsatisfiable. Then $B$ contains some introspective $\beta$, or there are $\alpha \in A, \beta \in B$ such that $\alpha \Rightarrow \beta$.

Proof. We prove the contraposition: suppose $B$ contains no introspective atom and there are no $\alpha \in A$ and $\beta \in B$ such that $\alpha \Rightarrow \beta$. We show that the state $s=A$ satisfies $\left(\bigwedge_{\alpha \in A} \alpha\right) \wedge\left(\bigwedge_{\beta \in B} \neg \beta\right)$. First, $s$ satisfies every element of $A$. Second, $s$ does not satisfy any $\beta \in B$ : otherwise $\beta$ would be introspective, or we would have $\alpha \Rightarrow \beta$ for some $\alpha \in A$.

Remark 1. When $\mathbb{A}$ is a singleton then $S_{i} p \wedge \neg J S p$ is satisfiable. While this anomaly could be taken care of by a modification of the semantics, we do not do so for the sake of readability and content ourselves with the observation that the $J S$ operator is superfluous when there is only one agent.

Remark 2. It would be interesting to generalise joint observability from $\mathbb{A}$ to arbitrary subsets of $\mathbb{A}$. For instance, after a gossiping phone call between agents $i$ and $j$ all secrets previously known by one of them become jointly observable by the group $\{i, j\}$. Such an extension was proposed in [33]; however, the solution turned out to be unsatisfactory. The problem is that joint visibilities of subgroups interact in complex ways, in particular when observability evolves. 
In the rest of the section we establish finite model property, given an axiomatisation, and prove NP-completeness of EL-O satisfiability.

\subsection{Finite model property}

A standard property of CPC is that $s \models^{\mathrm{CPC}} \varphi$ iff $s \cap \operatorname{Voc}(\varphi) \vDash^{\mathrm{CPC}} \varphi$. The non-standard truth condition for atoms in EL-O makes that this does not hold in EL-O. (It suffices to consider $s=\{J S p\}$ and $\varphi=S_{i} p$ to see this.) Notwithstanding, we obtain a finite model property by closing the state $s$.

First of all, let us say that two states $s$ and $s^{\prime}$ agree on the set of atoms $A \subseteq \mathrm{OA}$ when they assign the same truth value to every element of $A$, i.e., when for every $\alpha \in A, s \vDash \alpha$ iff $s^{\prime} \vDash \alpha$. This is not enough to guarantee that $s \cap A=s^{\prime} \cap A$. To witness, consider $s=\{J S p\}, s^{\prime}=\left\{S_{i} p\right\}$, and $A=\left\{S_{i} p\right\}: s$ and $s^{\prime}$ agree on $A$, but $s \cap A=\emptyset$ while $s^{\prime} \cap A=\left\{S_{i} p\right\}$. However by straightforward induction on the structure of formulas we get:

Proposition 4. Let $\varphi \in \mathcal{L}_{\text {bool }}(\mathrm{OA})$ be a formula and $s, s^{\prime} \in 2^{\mathrm{OA}}$ two states agreeing on $\operatorname{Voc}(\varphi)$. Then $s \vDash \varphi$ iff $s^{\prime} \vDash \varphi$.

Proposition 5. Let $\varphi \in \mathcal{L}_{\text {bool }}(\mathrm{OA})$ be a formula and $s \in 2^{\mathrm{OA}}$ a state. Then $s \vDash \varphi$ iff $(s \Rightarrow \cup \mathrm{I}-\mathrm{OA}) \cap \operatorname{Voc}(\varphi) \vDash \varphi$.

Proof. The proof is by induction on the structure of $\varphi$. The only non-trivial case is the induction base. We have $s=\alpha$ iff $s \Rightarrow \cup \mathrm{I}-\mathrm{OA} \vDash \alpha$ iff $(s \Rightarrow \cup \mathrm{I}-\mathrm{OA}) \cap$ $\operatorname{Voc}(\varphi) \vDash \alpha$. The last equivalence holds thanks to Proposition 4 .

\subsection{Complexity of EL-O satisfiability}

Proposition 6. Let $s \in 2^{\mathrm{OA}}$ be a state and $\varphi \in \mathcal{L}_{\text {bool }}(\mathrm{OA})$ a formula. Then $s \vDash \varphi$ iff $s \Rightarrow \mathrm{I}-\mathrm{OA} \vDash{ }^{\mathrm{CPC}} \varphi$.

Proof. The proof is by induction on the structure of $\varphi$. The only non-trivial case is the induction base. If $\alpha \in \mathrm{I}-\mathrm{OA}$ then both $s \vDash \alpha$ and I-OA ${ }^{\mathrm{CPC}} \alpha$ hold. Otherwise, for $\alpha \notin \mathrm{I}-\mathrm{OA}$ we have $s \vDash \alpha$ iff $\alpha \in s^{\Rightarrow}$ iff $s^{\Rightarrow} \vDash^{\mathrm{CPC}} \alpha$.

Proposition 7. The problem of deciding satisfiability of $\mathcal{L}_{\text {bool }}(\mathrm{OA})$ formulas is NP-complete. 


$\begin{array}{ll}S_{i} S_{i} \alpha & \left(\mathrm{Vis}_{1}\right) \\ J S J S \alpha & \left(\mathrm{Vis}_{2}\right) \\ J S S_{i} S_{i} \alpha & \left(\mathrm{Vis}_{3}\right) \\ J S \alpha \rightarrow S_{i} \alpha & \left(\mathrm{Vis}_{4}\right) \\ J S \alpha \rightarrow J S S_{i} \alpha & \left(\mathrm{Vis}_{5}\right)\end{array}$

Table 1: Axioms of EL-O

Proof. Hardness is the case because EL-O satisfiability and CPC satisfiability coincide for the $\mathcal{L}_{\text {bool }}(\mathbb{P})$ fragment of $\mathcal{L}_{\text {bool }}(\mathrm{OA})$ (Proposition 2). For membership, guess some subset $s \in 2^{\operatorname{Voc}(\varphi)}$ and check in polynomial time:

1. that $s$ contains all its relevant consequences: for every $\alpha \in \operatorname{Voc}(\varphi)$ and $\beta \in s$, check that if $\beta \Rightarrow \alpha$ then $\alpha \in s$;

2. that $s$ contains all introspective atoms from $\operatorname{Voc}(\varphi)$ : for every $\alpha \in$ $\operatorname{Voc}(\varphi)$, check that if $\alpha \in \mathrm{OA}$ then $\alpha \in s$;

3. that $s \models{ }^{\mathrm{CPC}} \varphi$.

Checking that $s \vDash^{\mathrm{CPC}} \varphi$ is tantamount to checking that $s \vDash \varphi$ because: (a) thanks to the first and second check, $s$ equals $(s \Rightarrow \cup \mathrm{I}-\mathrm{OA}) \cap \operatorname{Voc}(\varphi)$, and therefore $s \vDash^{\mathrm{CPC}} \varphi$ iff $(s \Rightarrow \cup \mathrm{I}-\mathrm{OA}) \cap \operatorname{Voc}(\varphi) \vDash^{\mathrm{CPC}} \varphi$; (b) in CPC, the latter is the same as $s \Rightarrow \mathrm{I}-\mathrm{OA} \vDash{ }^{\mathrm{CPC}} \varphi$ (contrarily to EL-O, in CPC irrelevant atoms outside $\operatorname{Voc}(\varphi)$ can be dropped from valuations); finally, (c) Proposition 6 applies.

\subsection{Axiomatisation of EL-O validities}

The EL-O validities of our language $\mathcal{L}_{\text {bool }}(\mathrm{OA})$ are axiomatised by the schemas of Table 1 together with CPC. We prove its completeness via CPC.

Proposition 8. Let $\varphi \in \mathcal{L}_{\text {bool }}(\mathrm{OA})$ be a formula. Then $\varphi$ is $\mathrm{EL}-\mathrm{O}$ valid iff $\varphi$ is provable in CPC from the axiom schemas Vis 1 Vis ${ }_{5}$ of Table 1. 
Proof. We again take advantage of Proposition 6 and show that $\operatorname{Vis}_{1} \sqrt{V_{i s_{5}}}$ characterise the set of introspectively closed states $\left\{s=\mathrm{I}-\mathrm{OA}: s \in 2^{\mathrm{OA}}\right\}$.

The right-to-left direction is clear: each of the five axiom schemas is valid in introspectively closed states. For the left-to-right direction, we show that every $s$ satisfying $V i s_{1}$ Vis $\sqrt{5}$ is introspectively closed.

For closure under atomic consequence, let $s \vDash \alpha$. The interesting case is when $\alpha=J S \alpha^{\prime}$. Then $s \vDash J S S_{i_{1}} \alpha^{\prime}$ by axiom Vis 5 , and also $s \vDash J S S_{i_{2}} S_{i_{1}} \alpha^{\prime}$, and so on: we can generate any $s \vDash J S S_{i_{m}} \ldots S_{i_{1}} \alpha^{\prime}$ and then, by Vis4, we can obtain $s \vDash S_{i_{m}} \ldots S_{i_{1}} \alpha^{\prime}$. Moreover, we have $s \vDash J S J S \alpha^{\prime}$ by Vis 2 , and in the same way, we can generate $s \vDash S_{i_{m}} \ldots S_{i_{1}} J S \alpha^{\prime}$ for any agents $i_{1}, \ldots i_{m}$ and $i$. We therefore obtain that $s$ satisfies any sequence whose strict postfix is $\alpha^{\prime}$, that is, every $\sigma \alpha^{\prime}$ for any $\sigma \in \mathrm{OBS}^{+}$.

We use the same technique to show that a state $s$ satisfying $\operatorname{Vis}_{1}+V i s_{5}$ satisfies every $\sigma S_{i} S_{i} \alpha$ for $\sigma \in \mathrm{OBS}^{*}$ and every $\sigma J S \alpha$ for $\sigma \in \mathrm{OBS}^{+}$: we obtain the first with $V i s_{1}$ (for $\sigma$ empty) and $V i s_{3}$, Vis, and $V i s_{5}$ (for $\sigma$ non-empty), and the second with $V i s_{2}, V i s_{4}$, and $V i s_{5}$,

Remark 3. We do not require states to satisfy the infinitary constraint "if $\sigma \alpha \in s$ for every $\sigma \in\left\{S_{i}: i \in \mathbb{A}\right\}^{+}$then $J S \alpha \in s$ ", which is the EL-O counterpart of the transitive closure constraint on Kripke models (see Remark4 4 in Section 4.2). The reason is technical: it is not obvious how to extend the axiomatisation of EL-O in a finitary way to account for that constraint. Our recent axiomatisation of 'common knowledge whether' of [34] might provide a solution; we however do not pursue this further here.

\section{EL-O as a fragment of epistemic logic S5}

We now situate our lightweight epistemic logic w.r.t. the standard epistemic logic S5 with 'knowing-that' operators $C K$ and $K_{i}$. We are going to exploit that $S_{i} \varphi$ can be viewed as an abbreviation of $K_{i} \varphi \vee K_{i} \neg \varphi$ and $J S \varphi$ as an abbreviation of $C K \varphi \vee C K \neg \varphi$. Then the language $\mathcal{L}_{\text {bool }}(\mathrm{OA})$ of EL-O becomes as a fragment of $\mathcal{L}_{\mathrm{EL}}$. When we make this identification we are going 
to talk about the EL-O fragment of $\mathcal{L}_{\mathrm{EL}}$.

Our main result is that for the EL-O fragment of $\mathcal{L}_{\mathrm{EL}}$, the axioms of Table 1 are sound and complete w.r.t. validity in S5 models (Proposition11). It follows by Proposition 7 that our lightweight epistemic logic is a fragment of standard epistemic logic with an NP-complete satisfiability problem. This is an important result because satisfiability of $\mathcal{L}_{\mathrm{EL}}$ formulas is PSPACE-complete as soon as there are two agents, and this is the case even without the common knowledge operator [35]. But first of all we recall the standard epistemic language $\mathcal{L}_{\mathrm{EL}}$ and the models in which its formulas are interpreted.

\subsection{The standard epistemic language}

The grammar of the standard language $\mathcal{L}_{\mathrm{EL}}$ is:

$$
\mathcal{L}_{\mathrm{EL}}: \varphi::=p|\neg \varphi| \varphi \wedge \varphi\left|K_{i} \varphi\right| C K \varphi
$$

where $p$ ranges over the set of propositional variables $\mathbb{P}$. The formula $K_{i} \varphi$ reads " $i$ knows that $\varphi$ " and $C K \varphi$ reads "it is common knowledge that $\varphi$ ". Hence there are no observability atoms: the vocabulary of $\mathcal{L}_{\mathrm{EL}}$ is just the set of propositional variables $\mathbb{P}$, of which we suppose that it is identical to the set $\mathbb{P}$ from which the observability atoms of Section 3.2 were built.

Example 4. The goal of the original gossip problem is to obtain shared knowledge of all secrets. This is expressed in the standard epistemic language $\mathcal{L}_{\mathrm{EL}}$ by $\bigwedge_{i, j \in \mathbb{A}}\left(K_{j} s_{i} \vee K_{j} \neg s_{i}\right)$. If we take $K_{i} \varphi$ to be an abbreviation of $p \wedge S_{i} p$ (see Section 2 then this goal reduces to the $\mathcal{L}_{\text {bool }}(\mathrm{OA})$ formula Goal $^{G_{1}}=$ $\bigwedge_{i, j \in \mathbb{A}} S_{j} s_{i}$. Shared knowledge up to depth 2 is expressed by $\bigwedge_{i, j, r \in \mathbb{A}}\left(K_{r} K_{j} s_{i} \vee\right.$ $\left.K_{r} K_{j} \neg s_{i}\right)$; reducing $K_{j}$ and $K_{r}$ we get $\bigwedge_{i, j, r \in \mathbb{A}}\left(S_{j} s_{i} \wedge S_{r} s_{i} \wedge S_{r} S_{j} s_{i}\right)$, which in CPC is equivalent to Goal $^{G_{2}}=\bigwedge_{i, j, r \in \mathbb{A}}\left(S_{j} s_{i} \wedge S_{r} S_{j} s_{i}\right)$. We have already seen these two $\mathcal{L}_{\text {bool }}(\mathrm{OA})$ formulas in Example 2. In the generalised gossip problem [6, 36] the goal is shared knowledge up to depth $k$, written $\bigwedge_{i \in \mathbb{A}} \bigwedge_{\vec{K} \in\left\{K_{j}: j \in \mathbb{A}\right\}^{k}}\left(\vec{K} s_{i} \vee\right.$ $\vec{K} \neg s_{i}$ ) in $\mathcal{L}_{\mathrm{EL}}$, where $\left\{K_{j}: j \in \mathbb{A}\right\}^{k}$ is the set of all sequences of individual 'knowing-that' operators of length $k$. It reduces to the $\mathcal{L}_{\text {bool }}(\mathrm{OA})$ formula 


$$
\text { Goal }^{G_{k}}=\bigwedge_{i \in \mathbb{A}} \bigwedge_{\sigma \in\left\{S_{j}: j \in \mathbb{A}\right\} \leq k} \sigma s_{i}
$$
operators up to length $k$.

\subsection{S5 Kripke models and the canonical model for EL-O}

We suppose that $C K$ and the $K_{i}$ are $\mathrm{S} 5$ modal operators and that common knowledge implies individual knowledge. Semantically, an S5 Kripke model [35] is a tuple $M=\left\langle W, \sim_{\mathbb{A}},\left\{\sim_{i}\right\}_{i \in \mathbb{A}}, V\right\rangle$ where $W$ is a nonempty set of possible worlds; $\sim_{\mathbb{A}}$ and every $\sim_{i}$ are equivalence relations on $W$ such that every $\sim_{i}$ is a subset of $\sim_{\mathbb{A}}$; and $V: W \longrightarrow 2^{\mathbb{P}}$ is a valuation. The interpretation of $\mathcal{L}_{\mathrm{EL}}$ formulas in a pointed Kripke model $(M, w)$ is:

$$
\begin{gathered}
M, w \vDash K_{i} \varphi \text { iff } M, w^{\prime} \vDash \varphi \text { for every } w^{\prime} \text { such that } w \sim_{i} w^{\prime} ; \\
M, w \vDash C K \varphi \text { iff } M, w^{\prime} \vDash \varphi \text { for every } w^{\prime} \text { such that } w \sim_{\mathbb{A}} w^{\prime} ;
\end{gathered}
$$

and as usual for the boolean operators. Formula $\varphi$ is valid in $M$ if $M, s \vDash \varphi$ for every $s \in W ; \varphi$ is valid in S5 Kripke models if $\varphi$ is valid in every S5 Kripke model.

Remark 4. Every $\sim_{i}$ being included in $\sim_{\mathbb{A}}$, the accessibility relation for common knowledge contains the transitive closure of the union of the individual accessibility relations $\left(\bigcup_{i \in \mathbb{A}} \sim_{i}\right)^{*}$, but does not necessarily equal it. The former property corresponds to the fact that in introspective states, $J S \alpha \in s$ implies that $\sigma \alpha \in s$ for any non-empty sequence $\sigma$ while the other direction fails to hold, as already discussed in Remark 3 . Our notion of common knowledge is therefore weaker than standard common knowledge in that the induction axiom $C K\left(\varphi \rightarrow \bigwedge_{i \in \mathbb{A}} K_{i} \varphi\right) \rightarrow(\varphi \rightarrow C K \varphi)$ is not valid. A similar notion was called 'fictitious knower' or 'any fool' by Genesereth and Nilsson [37] and was used in modal extensions of logic programming in the 90s [38, 39]. We have argued elsewhere that the induction axiom is too strong for a logic of common knowledge [40, 41].

Let us establish that every EL-O valuation $s$ can be identified with a particular pointed S5 Kripke model $\left(M^{\mathrm{EL}-\mathrm{O}}, s\right)$. The first thing we do is to define 
how the states' observability information determines equivalence relations between states. For every $s, s^{\prime} \in 2^{\mathrm{OA}}$ we define:

$$
\begin{aligned}
& s R_{i} s^{\prime} \text { iff } s \text { and } s^{\prime} \text { agree on every } \alpha \text { such that } s \vDash S_{i} \alpha ; \\
& s R_{\mathbb{A}} s^{\prime} \text { iff } s \text { and } s^{\prime} \text { agree on every } \alpha \text { such that } s \vDash J S \alpha .
\end{aligned}
$$

(Remember that agreement is defined in Section 3.5.) While these relations are clearly reflexive, it is not immediately clear that they are also symmetric and transitive; so let us prove that.

Proposition 9. All relations $R_{i}$ and $R_{\mathbb{A}}$ are equivalence relations.

Proof. We prove symmetry and transitivity of $R_{i}$ for an arbitrary $i$; the proof for $R_{\mathbb{A}}$ is analogous. For symmetry, suppose $s R_{i} s^{\prime}$. Then for every $\alpha$, if $s \vDash S_{i} \alpha$ then $s$ and $s^{\prime}$ agree on $\alpha$. Take an arbitrary $\beta$ such that $s^{\prime} \vDash S_{i} \beta$. $s^{\prime}$ agrees with $s$ on $S_{i} \beta$ because $s \vDash S_{i} S_{i} \beta$. Hence $s \vDash S_{i} \beta$, and $s$ and $s^{\prime}$ agree on $\beta$, and therefore $s^{\prime} R_{i} s$. For transitivity, suppose $s R_{i} s^{\prime}$ and $s^{\prime} R_{i} s^{\prime \prime}$ and suppose $s \vDash S_{i} \alpha$. We show that $s$ and $s^{\prime \prime}$ agree on $\alpha$. By hypothesis, $s$ and $s^{\prime}$ agree on $\alpha$. As observed above, $s$ and $s^{\prime}$ also agree on $S_{i} \alpha$ because $s \vDash S_{i} S_{i} \alpha$. Hence $s^{\prime} \vDash S_{i} \alpha$, and so $s^{\prime}$ and $s^{\prime \prime}$ agree on $\alpha$. Therefore $s$ and $s^{\prime \prime}$ agree on $\alpha$.

We are ready to define the canonical Kripke model for EL-O as the tuple $M^{\mathrm{EL}-\mathrm{O}}=\left\langle W^{\mathrm{EL}-\mathrm{O}}, \sim_{\mathbb{A}}^{\mathrm{EL}-\mathrm{O}},\left\{\sim_{i}^{\mathrm{EL}-\mathrm{O}}\right\}_{i \in \mathbb{A}}, V^{\mathrm{EL}-\mathrm{O}}\right\rangle$ with

$$
\begin{aligned}
W^{\mathrm{EL}-\mathrm{O}} & =I-S T A T E S=\left\{s^{\Rightarrow} \cup \mathrm{I}-\mathrm{OA}: s \in 2^{\mathrm{OA}}\right\}, \\
\sim_{\mathbb{A}}^{\mathrm{EL}-\mathrm{O}} & =R_{\mathbb{A}} \cap\left(W^{\mathrm{EL}-\mathrm{O}} \times W^{\mathrm{EL}-\mathrm{O}}\right), \\
\sim_{i}^{\mathrm{EL}-\mathrm{O}} & =R_{i} \cap\left(W^{\mathrm{EL}-\mathrm{O}} \times W^{\mathrm{EL}-\mathrm{O}}\right), \\
V^{\mathrm{EL}-\mathrm{O}}(w) & =w \cap \mathbb{P} \text { for every } w \in W^{\mathrm{EL}-\mathrm{O}} .
\end{aligned}
$$

Hence the possible worlds of $M^{\mathrm{EL}-\mathrm{O}}$ are the introspectively closed states I-STATES that we have defined in Section 3.3 .

Remark 5. In EL-O, the empty initial state $s_{0}=\emptyset$ models maximal ignorance: the agents do not know anything beyond tautologies. The simplicity of this modelling contrasts with the corresponding pointed Kripke model 
$\left(M^{\mathrm{EL}-\mathrm{O}}, s_{0}\right)$ : in that model, every agent can access infinitely many possible worlds from $s_{0}$. Actually the classical examples in introductory textbooks and articles about epistemic logic (such as the muddy children puzzle) are all modelled by finite Kripke models. As recently observed by Artemov [42], such finite models presuppose more or less tacitly a lot of common knowledge, which is too strong an assumption in many situations.

Proposition 10. Let $\varphi \in \mathcal{L}_{\text {bool }}(\mathrm{OA})$ and $s \in W^{\mathrm{EL}-\mathrm{O}}$. Then $s \vDash \varphi$ iff $M^{\mathrm{EL}-\mathrm{O}}, s \models \varphi$. Proof. The proof is by induction on the structure of $\varphi$. The only interesting case is the base case of atoms $\alpha \in \mathrm{OA}$. We use induction on the length of $\alpha$.

When $\alpha=p$ then $M, s \vDash p$ iff $s \vDash p$ for any $s \in W^{\mathrm{EL}-\mathrm{O}}$ by definition of $V^{\mathrm{EL}-\mathrm{O}}$.

When $\alpha=S_{i} \beta$ we prove the two directions of the equivalence.

- If $s=S_{i} \beta$ for some $\beta \in \mathrm{OA}$ and some $s \in W^{\mathrm{EL}-\mathrm{O}}$ : consider $s^{\prime} \in W^{\mathrm{EL}-\mathrm{O}}$ such that $s \sim_{i}^{\text {EL-O }} s^{\prime}$. By definition of $\sim_{i}^{\text {EL-O }}, s$ and $s^{\prime}$ agree on all $\alpha$ such that $s \vDash S_{i} \alpha$. Therefore if $s \vDash \beta$ then $s^{\prime} \vDash \beta$, and by the induction hypothesis, $M^{\mathrm{EL}-\mathrm{O}}, s^{\prime} \vDash \beta$; hence $M^{\mathrm{EL}-\mathrm{O}}, s \vDash K_{i} \beta$. If $s \vDash \neg \beta$, by the same argument, we have that $M^{\mathrm{EL}-\mathrm{O}}, s \vDash K_{i} \neg \beta$. Therefore $M^{\mathrm{EL}-\mathrm{O}}, s \vDash S_{i} \beta$.

- If $M^{\mathrm{EL}-\mathrm{O}}, s \vDash S_{i} \beta$ for some $\beta \in \mathrm{OA}$ and some $s \in W^{\mathrm{EL}-\mathrm{O}}$, then either $M^{\mathrm{EL}-\mathrm{O}}, s \vDash K_{i} \beta$ or $M^{\mathrm{EL}-\mathrm{O}}, s \vDash K_{i} \neg \beta$. Suppose that $s \not S_{i} \beta$. In particular $\beta \notin \mathrm{I}-\mathrm{OA}$. If $s \vDash \beta$, consider $s^{\prime}=s \backslash \beta^{\models}$, and if $s \not \models \beta$, consider $s^{\prime}=s \cup \beta^{\Rightarrow}$. In both cases $s^{\prime} \in W^{\mathrm{EL}-\mathrm{O}}$, and $s$ and $s^{\prime}$ agree on all $\alpha$ such that $s \vDash S_{i} \alpha$. Therefore $s \sim_{i}^{\mathrm{EL}-\mathrm{O}} s^{\prime}$, but by the induction hypothesis, $M^{\mathrm{EL}-\mathrm{O}}, s^{\prime} \vDash \beta$ iff $s^{\prime} \vDash \beta$ iff $s^{\prime} \vDash \neg \beta$ iff $M^{\mathrm{EL}-\mathrm{O}}, s \vDash \neg \beta$ : this contradicts the hypothesis that $M^{\mathrm{EL}-\mathrm{O}}, s \vDash S_{i} \beta$.

For the case $\alpha=J S \beta$ we proceed similarly.

\subsection{Completeness of the $\mathrm{EL}-\mathrm{O}$ fragment of $\mathcal{L}_{\mathrm{EL}}$ w.r.t. $\mathrm{S} 5$ validity}

We now show that the EL-O semantics in terms of observability and the standard Kripke semantics have the same validities as far as the EL-O fragment is concerned. 
Proposition 11. Let $\varphi \in \mathcal{L}_{\text {bool }}(\mathrm{OA})$ be a formula. Then $\varphi$ is valid in the S5 Kripke models of Section 4.2 iff $\varphi$ is provable in CPC from the EL-O axioms of Table 1

Proof. For soundness we show that the schemas $S_{i} S_{i} \alpha$, JS JS $\alpha$, JS $S_{i} S_{i} \alpha, J S \alpha \rightarrow$ $S_{i} \alpha, J S \alpha \rightarrow J S S_{i} \alpha$ are valid in S5 Kripke models. For the first it suffices to observe that $S_{i} S_{i} \alpha$ is equivalent to $K_{i}\left(K_{i} \alpha \vee K_{i} \neg \alpha\right) \vee K_{i} \neg\left(K_{i} \alpha \vee K_{i} \neg \alpha\right)$. The latter is equivalent in S5 to the propositionally valid $K_{i} \alpha \vee K_{i} \neg \alpha \vee \neg\left(K_{i} \alpha \vee K_{i} \neg \alpha\right)$. Validity of the other axiom schemas can be proved in a similar manner.

For completeness suppose that $\varphi$ is not provable from the EL-O axioms. By Proposition 8 there exists $s \subseteq$ OA such that $s \not \varphi$. Hence $s \Rightarrow \cup \mathrm{I}-\mathrm{OA} \not \not^{\mathrm{CPC}} \varphi$ by Proposition 6 and, as $s^{\Rightarrow} \cup \mathrm{I}-\mathrm{OA} \in W^{\mathrm{EL}-\mathrm{O}}, M^{\mathrm{EL}-\mathrm{O}}, s^{\Rightarrow} \cup \mathrm{I}-\mathrm{OA} \not \neq \varphi$ by Proposition 10. Hence $\varphi$ cannot be valid in S5 Kripke models.

\subsection{Axiomatisation of the $M^{\mathrm{EL}-\mathrm{O}}$ validities in $\mathcal{L}_{\mathrm{EL}}$}

It follows from Proposition 11 that in order to check satisfiability in S5 Kripke models of a formula in the EL-O fragment it suffices to take the canonical Kripke model $M^{\mathrm{EL}-\mathrm{O}}$ and go through its states. In other words, there is no formula in the EL-O fragment that is valid in $M^{\mathrm{EL}-\mathrm{O}}$ without being valid in the set of all S5 Kripke models. This fails to hold for the full language $\mathcal{L}_{\mathrm{EL}}$. This can be seem from the axiomatisation of the $\mathcal{L}_{\mathrm{EL}}$ validities in $M^{\mathrm{EL}-\mathrm{O}}$ of [43] and [9, Chapter 2] $]^{2}$ It is made up of: the EL-O axioms of Table 1, the axioms

$$
\begin{aligned}
K_{i} \alpha & \leftrightarrow \alpha \wedge S_{i} \alpha, & K_{i} \neg \alpha \leftrightarrow \neg \alpha \wedge S_{i} \alpha, \\
C K \alpha & \leftrightarrow \alpha \wedge J S \alpha, & C K \neg \alpha \leftrightarrow \neg \alpha \wedge J S \alpha
\end{aligned}
$$

\footnotetext{
${ }^{2}$ Our semantics differs from the semantics in these papers in that it is finitary. The only thing that changes is the soundness proof, which is routine.
} 
relating $K_{i}$ to $S_{i}$ and $C K$ to $J S$; all S5 principles for $K_{i}$ and $C K$; plus two axioms distributing $K_{i}$ and $C K$ over clauses:

$$
\begin{gathered}
K_{i}\left(\bigvee_{\alpha \in A^{+}} \alpha \vee \bigvee_{\alpha \in A^{-}} \neg \alpha\right) \leftrightarrow \begin{cases}\left(\bigvee_{\alpha \in A^{+}} K_{i} \alpha\right) \vee\left(\bigvee_{\alpha \in A^{-}} K_{i} \neg \alpha\right) & \text { if }\left(A^{+}\right)^{\models} \cap A^{-}=\emptyset, \\
\top & \text { otherwise; }\end{cases} \\
C K\left(\bigvee_{\alpha \in A^{+}} \alpha \vee \bigvee_{\alpha \in A^{-}} \neg \alpha\right) \leftrightarrow \begin{cases}\left(\bigvee_{\alpha \in A^{+}} C K \alpha\right) \vee\left(\bigvee_{\alpha \in A^{-}} C K \neg \alpha\right) & \text { if }\left(A^{+}\right)^{\models} \cap A^{-}=\emptyset, \\
\top & \text { otherwise. }\end{cases}
\end{gathered}
$$

The last two axioms resort to a generalisation of the 'caused-by' function from atoms to sets of atoms $A \subseteq \mathrm{OA}$, in the obvious way: $A^{\models}=\bigcup_{\alpha \in A} \alpha^{\Leftarrow}$. They are specific to observability-based knowledge and are typically invalid in normal modal logics. An instance of the first one is $K_{i}(p \vee q) \leftrightarrow\left(K_{i} p \vee K_{i} q\right)$, for different $p$ and $q$. This is a strong principle: to give an example, if it is known that the butler or the gardener was the murderer then it is also known who of them it was. The reason is that our logic is built on atomic observability information: what is modelled in $M^{\mathrm{EL}-\mathrm{O}}$ are forms of individual and common knowledge that are respectively obtained via individual observation and joint observation of facts. This differs conceptually from the classical operators of individual and common knowledge as studied in epistemic logic [12]. The property of distribution over falsifiable disjunctions of literals is common in epistemic logics that are based on observability of propositional variables. It is shown in [29] that it can be avoided if one introduces new propositional variables.

The axiomatics allows us to reduce every $\mathcal{L}_{\mathrm{EL}}$ formula to an equivalent $\mathcal{L}_{\text {bool }}(\mathrm{OA})$ formula. For example, positive introspection $K_{i} p \rightarrow K_{i} K_{i} p$ reduces to $\left(p \wedge S_{i} p\right) \rightarrow\left(p \wedge S_{i} p \wedge S_{i} S_{i} p\right)$. Observe that the latter is EL-O valid.

\section{Epistemic planning with conditional effects}

We now define actions and planning tasks within our framework. We assume deterministic actions with conditional effects whose preconditions 
are described by EL-O formulas and whose positive and negative effects are sets of observability atoms. We have already argued in Section 2 that conditional effects are important in epistemic planning: the effects of an action on agent $i$ 's epistemic state typically depend on whether $i$ sees the variables that are modified by the action or not.

We start by defining classical planning tasks [44] and then introduce our simple epistemic planning tasks. The name 'simple' will be justified by our translation to classical planning of Section 5.5 .

\subsection{Action descriptions and planning tasks}

Just as for the definition of boolean formulas in Section 3.1, we consider an abstract vocabulary $\mathbb{V}$ with which actions are described. An action description over $\mathbb{V}$ (or action for short) is a pair $a=\langle p r e(a)$,eff(a) $\rangle$ where $\operatorname{pre}(\mathrm{a}) \in \mathcal{L}_{\text {bool }}(\mathbb{V})$ and $\operatorname{eff}(\mathrm{a}) \subseteq \mathcal{L}_{\text {bool }}(\mathbb{V}) \times 2^{\mathbb{V}} \times 2^{\mathbb{V}}$. The formula pre(a) is the precondition of a, describing when the action may be applied, and eff (a) are the conditional effects of a, describing which atomic formulas the action may add or remove from the current state under additional conditions. For each conditional effect

$$
c e=\left\langle\operatorname{cnd}(c e), c e f f^{+}(c e), c e f f^{-}(c e)\right\rangle
$$

in eff(a), $c n d(c e)$ is the condition of $c e, c e f f^{+}(c e)$ are the added and $c e f f^{-}(c e)$ are the deleted atomic formulas. The vocabulary and the length of an action description are defined by:

$$
\begin{aligned}
\operatorname{Voc}(\mathrm{a}) & =\operatorname{Voc}(\operatorname{pre}(\mathrm{a})) \cup \bigcup_{c e \in e f f(\mathrm{a})}\left(\operatorname{Voc}(\operatorname{cnd}(c e)) \cup c e f f^{+}(c e) \cup c^{\prime} e f f^{-}(c e)\right) ; \\
\ell(\mathrm{a}) & =\ell(\operatorname{pre}(\mathrm{a}))+\sum_{c e \in e f f(\mathrm{a})}\left(\ell(\operatorname{cnd}(c e))+\left(\sum_{p \in c e f f^{+}(c e)} \ell(p)\right)+\left(\sum_{p \in c e f f^{-}(c e)} \ell(p)\right)\right) .
\end{aligned}
$$

Remember that each $p \in \mathbb{V}$ has its length $\ell(p) \geq 1$, for some integer.

A planning task over $\mathbb{V}$ is a tuple $\mathcal{P}=\left\langle\mathbb{V}\right.$, Act, $s_{0}$, Goal $\rangle$ where $\mathbb{V}$ is some vocabulary, Act is a set of action descriptions, $s_{0} \in 2^{\mathbb{V}}$ is the initial state, and 
Goal $\in \mathcal{L}_{\text {bool }}(\mathbb{V})$ is the goal formula. Its relevant vocabulary and length are:

$$
\begin{aligned}
\operatorname{Voc}(\mathcal{P}) & =\left(\bigcup_{\mathrm{a} \in A c t} \operatorname{Voc}(\mathrm{a})\right) \cup s_{0} \cup \operatorname{Voc}(\text { Goal }) ; \\
\ell(\mathcal{P}) & =\left|s_{0}\right|+\ell(\text { Goal })+\sum_{\mathrm{a} \in A c t} \ell(\mathrm{a}) .
\end{aligned}
$$

The solutions of a planning task depend on the interpretation of formulas and actions. We have already seen the classical EL-O interpretation of formulas (Section 3.1) and the EL-O interpretation of formulas (Section

520 3.4. As to actions, we are going to define their classical and their EL-O interpretation. Both are relations on the set of states $2^{\mathbb{V}}$. Given some set of action-interpreting relations $\mathcal{R}$, we say that the state $s$ is reachable from the state $s_{0}$ via $\mathcal{R}$ if there exists an integer $m \geq 0$, a sequence of states $t_{0}, \ldots, t_{m}$ from $2^{\mathbb{V}}$, and a sequence of relations $R_{1}, \ldots, R_{m}$ from $\mathcal{R}$ such that $s_{0}=t_{0}$, ${ }_{525} s=t_{m}$, and $t_{k-1} R_{k} t_{k}$ for every $k$ such that $1 \leq k \leq m$. Solvability of a planning task amounts to reachability of a state satisfying the goal from the initial state $s_{0}$ via the relations interpreting the actions.

\subsection{Classical planning}

When an action description a has a precondition and two (not necessarily different) conditional effects whose preconditions are jointly satisfiable with pre(a) then there is a conflict. The other way round, we say that a is classically consistent if and only if for every $c e_{1}, c e_{2} \in$ eff(a), if $\operatorname{pre}(\mathrm{a}) \wedge \operatorname{cnd}\left(c e_{1}\right) \wedge \operatorname{cnd}\left(c e_{2}\right)$ is CPC satisfiable then $\operatorname{ceff}^{+}\left(c e_{1}\right)$ and $c e f f^{-}\left(c e_{2}\right)$ are disjoint.

535 Example 5. For an example of inconsistency consider the following description of the action $\operatorname{swap}_{p, q}$ swapping the truth values of $p$ and $q$. Suppose this action can always be executed, so its precondition is $\operatorname{pre}\left(\operatorname{swap}_{p, q}\right)=\mathrm{T}$, and suppose its conditional effects are naively described by eff $\left(\operatorname{swap}_{p, q}\right)=$ $\{\langle p,\{q\},\{p\}\rangle,\langle q,\{p\},\{q\}\rangle\}$. These two conditional effects conflict because $\top \wedge p \wedge q$ 540 is CPC-satisfiable. Observe that we can make the description classically 
consistent by replacing the precondition by $\neg(p \leftrightarrow q)$ : the action is executable only if the truth values of $p$ and $q$ differ.

A classically consistent action description determines the following relation on the set of states $2^{\mathrm{V}}$ :

$$
s R_{\mathrm{a}}^{\mathrm{CPC}} s^{\prime} \text { iff } s \vDash^{\mathrm{CPC}} \operatorname{pre}(\mathrm{a}) \text { and } s^{\prime}=\left(s \backslash \bigcup_{\substack{c e \in e f f(\mathrm{a}), s \models^{\mathrm{CPC}} \text { cnd (ce) }}} \operatorname{ceff} f^{-}(c e)\right) \cup \bigcup_{\substack{c e \in e f f(\mathrm{a}), s \models^{\mathrm{CPC}} \text { cnd (ce) }}} c e f f^{+}(c e) \text {. }
$$

A planning task is classically solvable if a state satisfying the goal formula is reachable from the initial state via $\left\{R_{\mathrm{a}}^{\mathrm{CPC}}: \mathrm{a} \in A c t\right\}$, i.e., via the CPC-interpretation of the actions in Act. It is known that classical solvability of a planning task is a PSPACE-complete reasoning problem [14].

\subsection{EL-O consistent action descriptions}

In order to describe epistemic preconditions and effects we instantiate the vocabulary $\mathbb{V}$ by the set of observability atoms $O A$. An action description $\mathrm{a}=\langle\operatorname{pre}(\mathrm{a}), e f f(\mathrm{a})\rangle$ over OA is EL-O consistent if and only if

1. for every ce $\in$ eff(a), ceff-(ce) contains no introspective atoms;

2. for every $c e_{1}, c e_{2} \in e f f(\mathrm{a})$, if $\operatorname{pre}(\mathrm{a}) \wedge c n d\left(c e_{1}\right) \wedge c n d\left(c e_{2}\right)$ is satisfiable in EL-O then $c e f f^{+}\left(c e_{1}\right)$ and $\left(c e f f^{-}\left(c e_{2}\right)\right)^{\models}$ are disjoint.

Proposition 3 provides formal support to these two conditions. Let us ex-

plain the intuitions behind them. The first is clear: it makes no sense to delete introspective atoms. The second condition requires that two conditional effects of an action cannot conflict when pre(a) and their triggering conditions are jointly satisfiable. Hence when an action a has conditional effects $c e_{1}, c e_{2} \in e f f(a)$ and there are $\alpha_{1} \in \operatorname{ceff}^{+}\left(c e_{1}\right)$ and $\alpha_{2} \in \operatorname{ceff}^{-}\left(c e_{2}\right)$ such that $\alpha_{1} \Rightarrow \alpha_{2}$ then $\operatorname{pre}(\mathrm{a}) \wedge \operatorname{cnd}\left(c e_{1}\right) \wedge \operatorname{cnd}\left(c e_{2}\right)$ must be inconsistent. Note that it follows from the transitivity of introspective consequence $\Rightarrow$ that $c e f f^{+}\left(c e_{1}\right)$ and $\left(c e f f^{-}\left(c e_{2}\right)\right)^{\Leftarrow}$ are disjoint iff $c e f f^{+}\left(c e_{1}\right) \Rightarrow$ and $\left(c e f f^{-}\left(c e_{2}\right)\right)^{\Leftarrow}$ are.

Example 6. The action flip of flipping a light switch is described by pre(flip) $=$ $T$ and eff(flip) $=\{\langle$ On, $\emptyset,\{$ On $\}\rangle,\langle\neg$ On, $\{$ On $\}, \emptyset\rangle\}$. It is EL-O consistent: first, none 
of the negative effects is an introspective atom, and second, although there are two conditional effects with contradictory add- and delete-lists, there can be no conflict because the conjunction $\top \wedge$ On $\wedge \neg$ On of the precondition and the conditions of the two conditional effects is unsatisfiable.

Example 7. Let us describe calls in the original gossip problem where the goal is to obtain shared knowledge of depth $k=1$. Suppose OA is built from $\mathbb{A}=\{1, \ldots, n\}$ and $\mathbb{P}=\left\{s_{i}: i \in \mathbb{A}\right\}$. During the action call ${ }_{j}^{i}$, in which a call is made between agents $i$ and $j$ (this action being symmetrical), the two agents tell each other every secret they know among all $n$ secrets. We have call ${ }_{j}^{i}=\left\langle\operatorname{pre}\left(\right.\right.$ call $\left._{j}^{i}\right), e f f\left(\right.$ call $\left.\left._{j}^{i}\right)\right\rangle$ with $\operatorname{pre}\left(\right.$ call $\left._{j}^{i}\right)=\mathrm{T}$ and:

$$
\begin{aligned}
e f f\left(\text { call }_{j}^{i}\right)= & \left\{\left\langle S_{i} s_{1} \vee S_{j} s_{1},\left\{S_{i} s_{1}, S_{j} s_{1}\right\}, \emptyset\right\rangle,\right. \\
& \ldots, \\
& \left.\left\langle S_{i} s_{n} \vee S_{j} s_{n},\left\{S_{i} s_{n}, S_{j} s_{n}\right\}, \emptyset\right\rangle\right\} .
\end{aligned}
$$

Intuitively, a secret becomes observable for both agents if at least one of them observes it. Each call ${ }_{j}^{i}$ is EL-O consistent because it has no negative effects. The length of a call is $\ell\left(\right.$ call $\left._{j}^{i}\right)=5+(|\mathbb{A}| \times(8+4+0))=12 n+5$. (Remember that disjunctions $S_{i} s_{k} \vee S_{j} s_{k}$ abbreviate $\neg\left(\neg S_{i} s_{1} \wedge \neg S_{j} s_{1}\right)$.)

Here is a more complex example of a consistent action.

Example 8. Consider the action pour of pouring a bottle of liquid on a healthy lawn [45]. Factually, its effect is that if the liquid is poisonous the lawn becomes dead. Epistemically, pour has several conditional effects: (1) if $i$ knows that Poisonous then $i$ will know that the lawn is dead; (2) if $i$ does not know whether Poisonous and if the lawn is known to be healthy ${ }^{3}$ then $i$ will no longer know whether the lawn is healthy or dead. (There is a third possible epistemic situation where $i$ knows that $\neg$ Poisonous, but we

\footnotetext{
${ }^{3}$ In [45], the condition of the last effect is only that it is not known that $\neg$ Poisonous, and the effect is that it is no longer known that $\neg$ Dead. This however produces an unintuitive result in states where the liquid is known to be poisonous.
} 
need not say anything here because there is no effect as far as the status of Dead is concerned.) Consider the description of pour with precondition $\operatorname{pre}($ pour $)=T$ and effect

$$
\begin{aligned}
\text { eff }(\text { pour })= & \{\langle\text { Poisonous, }\{\text { Dead }\}, \emptyset\rangle, \\
& \left\langle\text { Poisonous } \wedge S_{i} \text { Poisonous, }\left\{S_{i} \text { Dead }\right\}, \emptyset\right\rangle, \\
& \left.\left\langle\neg S_{i} \text { Poisonous } \wedge \neg \text { Dead } \wedge S_{i} \text { Dead, } \emptyset,\left\{S_{i} \text { Dead }\right\}\right\rangle\right\} .
\end{aligned}
$$

The first conditional effect is factual and the last two are epistemic. The second represents (1): Poisonous $\wedge S_{i}$ Poisonous expresses that $i$ knows that the liquid is poisonous; and the third represents (2): $\neg$ Dead $\wedge S_{i}$ Dead expresses that $i$ knows that the lawn is healthy. This action description is ELO consistent in particular because the conjunction of the two last conditions Poisonous $\wedge S_{i}$ Poisonous and $\neg S_{i}$ Poisonous $\wedge \neg$ Dead $\wedge S_{i}$ Dead is inconsistent.

\subsection{Simple epistemic planning tasks}

Every EL-O consistent action a determines a relation $R_{\mathrm{a}}^{\mathrm{EL}-\mathrm{O}}$ between states that is a partial function: for every $s, s^{\prime} \in 2^{\mathrm{OA}}$,

$$
s R_{\mathrm{a}}^{\mathrm{EL}-\mathrm{O}} s^{\prime} \quad \text { iff } s \vDash \operatorname{pre}(\mathrm{a}) \text { and } s^{\prime}=\left(s \backslash \bigcup_{\substack{c \in \in e f f(\mathrm{a}), s \models c n d(c e)}}\left(c e f f^{-}(c e)\right)^{\Leftarrow}\right) \cup \bigcup_{\substack{c e \in e f f(\mathrm{a}), s \models c n d(c e)}}\left(c e f f f^{+}(c e)\right)^{\Rightarrow} \text {. }
$$

That is, if its precondition is satisfied then an action

- removes negative effects of firing conditional effects, plus their causes;

- adds positive effects of firing conditional effects, plus their consequences.

Thanks to EL-O consistency the order of removals and additions does not matter. Moreover, if we start from an introspectively closed state in I-STATES then the resulting state is also introspectively closed.

A simple epistemic planning task is a planning task over some subset of the set of observability atoms OA. It is EL-O solvable iff there is a state $s$ such that $s \vDash$ Goal that is reachable from $s_{0}^{\Rightarrow} \cup$ I-OA via the EL-Ointerpretation of the actions in $A c t$, i.e., via $\left\{R_{\mathrm{a}}^{\mathrm{EL}-\mathrm{O}}: \mathrm{a} \in A c t\right\}$. 
Example 9. The planning task corresponding to the original gossip problem is $G_{1}=\left\langle\mathrm{OA}, A c t^{G_{1}}, s_{0}^{G_{1}}\right.$, Goal $\left.^{G_{1}}\right\rangle$ with

$$
\begin{aligned}
A c t^{G_{1}} & =\left\{\text { call }_{j}^{i}: i, j \in \mathbb{A} \text { and } i \neq j\right\}, \\
s_{0}^{G_{1}} & =\left\{S_{i} s_{i}: i \in \mathbb{A}\right\} \cup S \text { for some } S \subseteq\left\{s_{i}: i \in \mathbb{A}\right\}, \\
\text { Goal }^{G_{1}} & =\bigwedge_{i, j \in \mathbb{A}} S_{i} s_{j} .
\end{aligned}
$$

It can be solved via $2 n-4$ calls; when $n \geq 4$ then there is no EL-O solution that takes less calls [46, 47, 48]. For instance, for $n=4$ the sequence call $_{2}^{1}$; call ${ }_{4}^{3}$; call ${ }_{3}^{1}$; call ${ }_{4}^{2}$ is an EL-O solution with 4 calls.

If the vocabulary is $\mathbb{P}$ then classical planning tasks as defined in Section 5.2 are particular simple epistemic planning tasks: when action descriptions contain no observability operators then classical consistency and EL-O consistency of actions coincide; moreover, ceff $f^{-}(c e)=\left(c e f f^{-}(c e)\right)^{\models}$ and $c e f f^{+}(c e)=\left(c e f f^{+}(c e)\right) \Rightarrow$ for all conditional effects, and therefore $R_{\mathrm{a}}^{\mathrm{EL}-\mathrm{O}}=R_{\mathrm{a}}^{\mathrm{CPC}}$ for every action a. It follows from PSPACE hardness of classical solvability that EL-O solvability of simple epistemic planning tasks is PSPACE-hard, too.

\subsection{Translation into classical planning}

The expansion of a simple epistemic planning task $\mathcal{P}=\left\langle\mathrm{OA}\right.$, Act, $s_{0}$, Goal $\rangle$ adds 'enough' consequences of atoms, viz. the relevant ones occurring in $\mathcal{P}$ :

$$
\operatorname{Exp}(\mathcal{P})=\left\langle\operatorname{Voc}(\mathcal{P}),\left\{\left\langle\operatorname{pre}(\mathrm{a}), \operatorname{Exp}_{\mathcal{P}}(e f f(\mathrm{a}))\right\rangle: \mathrm{a} \in \operatorname{Act}\right\},\left(s_{0}^{\Rightarrow} \cup \mathrm{I}-\mathrm{OA}\right) \cap \operatorname{Voc}(\mathcal{P}), \text { Goal }\right\rangle
$$

where the expansion of the effects of an action relative to $\mathcal{P}$ is defined as:

$$
\operatorname{Exp}_{\mathcal{P}}(e f f(\mathrm{a}))=\left\{\left\langle\operatorname{cnd}(c e), c e f f^{+}(c e)^{\Rightarrow} \cap \operatorname{Voc}(\mathcal{P}), \operatorname{ceff}{ }^{-}(c e)^{\Leftarrow} \cap \operatorname{Voc}(\mathcal{P})\right\rangle: c e \in e f f(\mathrm{a})\right\} .
$$

After expansion classical solvability and EL-O solvability coincide:

Proposition 12. Let $\mathcal{P}=\left\langle\mathrm{OA}\right.$, Act, $s_{0}$, Goal $\rangle$ be a simple epistemic planning task. Then $\mathcal{P}$ is $\mathrm{EL}-\mathrm{O}$ solvable iff $\operatorname{Exp}(\mathcal{P})$ is classically solvable. 
Proof. We restrict the domain of the interpretation of actions to $I$-STATES $\left.\right|_{\operatorname{Voc}(\mathcal{P})}=\{s \cap \operatorname{Voc}(\mathcal{P}): s \in I-S T A T E S\}=\left\{\left(s^{\Rightarrow} \cup \mathrm{I}-\mathrm{OA}\right) \cap \operatorname{Voc}(\mathcal{P}): s \in 2^{\mathrm{OA}}\right\}$

For each a $\in$ Act we write $s R_{\mathrm{a}}^{\mathcal{P}} s^{\prime}$ for the restriction of $s R_{\mathrm{a}}^{\mathrm{EL}-\mathrm{O}} s^{\prime}$ to the set $I$-STATES $\left.\right|_{\operatorname{Voc}(\mathcal{P})}$. We therefore have $s R_{\mathrm{a}}^{\mathcal{P}} s^{\prime}$ iff $s, s^{\prime} \in I$-STATES $\left.\right|_{\operatorname{Voc}(\mathcal{P})}, s \vDash \operatorname{pre}(\mathrm{a})$ and

$$
s^{\prime}=\left(s \backslash \bigcup_{\substack{c e \in e f f(a), s \models \operatorname{cnd}(c e)}}\left(c e f f^{-}(c e)\right)^{\models} \cap \operatorname{Voc}(\mathcal{P})\right) \cup \bigcup_{\substack{c e \in e f f(a), s \notin \operatorname{cnd}(c e)}}\left(c e f f^{+}(c e)\right)^{\Rightarrow} \cap \operatorname{Voc}(\mathcal{P}) .
$$

It is easily shown that if $s \in I-\left.S T A T E S\right|_{\operatorname{Voc}(\mathcal{P})}$ then $s^{\prime} \in I-\left.S T A T E S\right|_{\operatorname{Voc}(\mathcal{P})}$.

We now show the following result: for any state $s \in I$-STATES and action a $\in A c t$, there exists a state $s^{\prime} \in I$-STATES such that $s R_{\mathrm{a}}^{\mathrm{EL}-\mathrm{O}} s^{\prime}$ iff there exists a state $s^{\prime \prime} \in I-\left.S T A T E S\right|_{\operatorname{Voc}(\mathcal{P})}$ such that $(s \cap \operatorname{Voc}(\mathcal{P})) R_{\mathrm{a}}^{\mathcal{P}} s^{\prime \prime}$, and in that case we have that $s^{\prime \prime}=s^{\prime} \cap \operatorname{Voc}(\mathcal{P})$. The first half is straightforward: as $\operatorname{Voc}($ pre $(\mathrm{a})) \subseteq$ $\operatorname{Voc}(\mathcal{P})$, for any introspectively closed state $s, s$ and $s \cap \operatorname{Voc}(\mathcal{P})$ agree on pre(a) and hence $s \vDash \operatorname{pre}(\mathrm{a})$ iff $s \cap \operatorname{Voc}(\mathcal{P}) \vDash \operatorname{pre}(\mathrm{a})$ (following Proposition 4). By the same argument, if $s R_{\mathrm{a}}^{\mathrm{EL}-\mathrm{O}} s^{\prime}$ and if $(s \cap \operatorname{Voc}(\mathcal{P})) R_{\mathrm{a}}^{\mathcal{P}} s^{\prime \prime}$, then

$$
s^{\prime}=\left(s \backslash \bigcup_{\substack{c e \in e f f(a), s \models c n d(c e)}}\left(c e f f^{-}(c e)\right) \in\right) \cup \bigcup_{\substack{c e \in e f f(a), s \models c n d(c e)}}\left(c e f f^{+}(c e)\right) \Rightarrow
$$

and therefore

$$
s^{\prime}=\left(s \backslash \bigcup_{\begin{array}{c}
c e \in e f f(a), \\
s \cap \operatorname{Voc}(\mathcal{P}) \models \operatorname{cnd}(c e)
\end{array}}\left(c e f f^{-}(c e)\right)=\right) \cup \bigcup_{\begin{array}{c}
c e \in e f f(a), \\
s \cap \operatorname{Voc}(\mathcal{P}) \models \operatorname{cnd}(c e)
\end{array}}\left(c e f f^{+}(c e)\right)^{\Rightarrow} .
$$

By intersecting this with $\operatorname{Voc}(\mathcal{P})$ we conclude that $s^{\prime} \cap \operatorname{Voc}(\mathcal{P})=s^{\prime \prime}$.

We can extend this result to any sequence of actions: for every $s \in$ $I$-STATES $\left.\right|_{\operatorname{Voc}(\mathcal{P})}$, there exists a state $s^{\prime}$ that is reachable from $s$ via $\left\{R_{\mathrm{a}}^{\mathrm{EL}-\mathrm{O}}\right.$ : $a \in A c t\}$ iff there exists a state $s^{\prime \prime}$ that is reachable from $s \cap \operatorname{Voc}(\mathcal{P})$ via $\left\{R_{\mathrm{a}}^{\mathcal{P}}: \mathrm{a} \in A c t\right\}$, and in that case $s^{\prime \prime}=s^{\prime} \cap \operatorname{Voc}(\mathcal{P})$. Moreover, $s^{\prime}$ and $s^{\prime \prime}$ agree on $\operatorname{Voc}(G o a l)$, and therefore $\mathcal{P}$ is EL-O solvable via $\left\{R_{\mathrm{a}}^{\mathrm{EL}-\mathrm{O}}:\right.$ a $\left.\in A c t\right\}$ 
iff the planning task $\left\langle\operatorname{Voc}(\mathcal{P}), A c t,\left(s_{0}^{\Rightarrow} \cup \mathrm{I}-\mathrm{OA}\right) \cap \operatorname{Voc}(\mathcal{P})\right.$, Goal $\rangle$ is EL-O solvable via $\left\{R_{\mathrm{a}}^{\mathcal{P}}: \mathrm{a} \in A c t\right\}$. Finally, thanks to Proposition 6 the latter is the case iff the expansion of $\mathcal{P}$ is classically solvable via $\left\{R_{\mathrm{a}}^{\mathrm{CPC}}: \mathrm{a} \in A c t\right\}$.

\subsection{Complexity}

Our translation from epistemic planning to classical planning increases task length only polynomially:

Proposition 13. For every simple epistemic planning task $\mathcal{P}, \ell(\operatorname{Exp}(\mathcal{P})) \leq$ $620 \quad(\ell(\mathcal{P}))^{2}$.

Proof. This follows from Proposition 1 .

Proposition 14. Solvability of a simple epistemic planing task is PSPACEcomplete.

Proof. We have seen in Section 5.2 that classical planning tasks can be viewed as simple epistemic planning tasks. The solvability problem is therefore PSPACE-hard just as classical planning [14]. Membership follows from Propositions 13 and 12 and from Bylander's proof that classical planning is in PSPACE [14].

\section{Applications}

${ }_{630}$ In this section, we give some examples of epistemic planning tasks. We start with the Byzantine Generals Problem and some toy examples most of which were introduced in [49]. We then focus on the generalised gossip problem.

\subsection{Two Generals’ Problem}

The Two Generals' Problem [50, 51] is about coordination by communicating over an unreliable channel, highlighting the importance of common knowledge. The Byzantine Generals' Problem [52] is a generalisation of that problem. In short, two generals need to coordinate an attack; they can 
win only if they attack at the same time: either both attack in the morning $(t a)$ or both attack in the afternoon $(\neg t a)$. To communicate they must send a messenger who can be captured. They must decide on a time to attack, agree on this time, and each general must know that the other has agreed, and so on. To do this, one general sends a time for the attack, but since he cannot be sure the message was received, the other general must send an acknowledgment. Of course, just like the original message, the acknowledgment can be lost, so the first general must send an acknowledgment, and so on. It is impossible to be fully coordinated, i.e., to obtain common knowledge of the fact that the second general knows the time of the attack.

Formally, with $\mathbb{A}=\{1,2\}$ and $\mathbb{P}=\{t a\}$, we define the atom $\alpha_{n}$ as:

- $\alpha_{n}=\underbrace{S_{1} S_{2} S_{1} \ldots S_{2} \text { ta }}_{n \text { alternations }}$ if $n$ is even;

- $\alpha_{n}=\underbrace{S_{2} S_{1} S_{2} \ldots S_{2} \text { ta }}_{n \text { alternations }}$ if $n$ is odd.

For $n \geq 0$ we define the family of actions sndMsg ${ }_{n}$ by stipulating:

$$
\begin{aligned}
\text { pre }\left(\text { sndMsg }_{n}\right) & =t a \wedge S_{1} t a \wedge S_{2} t a \wedge \alpha_{2} \wedge \alpha_{3} \wedge \cdots \wedge \alpha_{n-1} \\
\text { eff }\left(\text { sndMsg }_{n}\right) & =\left\{\left\langle\top,\left\{t a, S_{1} \text { ta }, S_{2} \text { ta }, \alpha_{2}, \alpha_{3}, \ldots, \alpha_{n}\right\}, \emptyset\right\rangle\right\}
\end{aligned}
$$

whose effect is that the time of the attack is set, that both agents know it, that 1 knows that 2 knows it, and so on until $n$.

The planning task to be solved is $\mathcal{P}=\left\langle\mathrm{OA}\right.$, Act, $s_{0}$, Goal $\rangle$ with $s_{0}=\emptyset$, Goal $=$ 655 $J S S_{2} t a$, and Act $=\left\{\right.$ sndMsg $\left._{n}: n \geq 0\right\}$. There is no solution for this task: while all sequences $\left\langle\right.$ sndMsg $_{0}, \ldots$, sndMsg $\left._{n}\right\rangle$ are executable, none of them reaches a state where $J S S_{2} t a$ is true.

Remark 6. The initial state $s_{0}=\emptyset$ of the Two Generals' Problem illustrates a case of maximal ignorance as discussed in Remark 5 . The infiniteness of the maximal ignorance Kripke model may explain why the DEL literature pays only little attention to this and other Byzantine coordination problems: most presentations are in semantical terms and use model checking 


\begin{tabular}{|c|c|c|}
\hline action & precondition & conditional effects \\
\hline enter $_{i}$ & $\neg \ln _{i}$ & $\left\{\left\langle T,\left\{\ln _{i}\right\}, \emptyset\right\rangle\right\}$ \\
\hline enter ${ }_{i}^{\prime}$ & $\neg \ln _{i} \wedge \neg \ln _{j}, i \neq j$ & $\left\{\left\langle\mathrm{~T},\left\{\ln _{i}\right\}, \emptyset\right\rangle\right\}$ \\
\hline leave $_{i}$ & $\ln _{i}$ & $\left\{\left\langle T, \emptyset,\left\{\ln _{i}\right\}\right\rangle\right\}$ \\
\hline reveal $_{i}$ & $\ln _{i}$ & $\left\{\left\langle\top,\left\{S_{i} m\right\}, \emptyset\right\rangle\right\} \cup$ \\
\hline & & $\left\{\left\langle\mathrm{In}_{j},\{J S m\}, \emptyset\right\rangle, j \neq i\right\}$ \\
\hline informs $_{1,2} m$ & $\left(\ln _{1} \leftrightarrow \ln _{2}\right) \wedge S_{1} m \wedge \neg\left(S_{2} m \wedge S_{1} S_{2} m\right)$ & $\{\langle\top,\{J S m\}, \emptyset\rangle\}$ \\
\hline informs $s_{1,2}^{\prime} m$ & $\left(\ln _{1} \leftrightarrow \ln _{2}\right) \wedge S_{1} m \wedge \neg S_{2} m \wedge S_{1} S_{2} m$ & $\{\langle\top,\{J S m\}, \emptyset\rangle\}$ \\
\hline
\end{tabular}

Table 2: Action descriptions for the message task

in finite Kripke models. (A finite modelling was however proposed recently in [19].)

\subsection{Learning a message}

Suppose two agents 1 and 2 are outside a room $\left(\neg \mathrm{In}_{1} \wedge \neg \mathrm{In}_{2}\right)$. The room contains a message that we suppose for simplicity to be the value of the propositional variable $m$. Each of the agents can enter and leave the room and can (temporarily) reveal the message when she is in the room. In case the other agent is also in the room the message is jointly seen. (So this is a conditional effect.) We define a series of planning tasks where the initial state is $s_{0}=\{m\}$ and where we vary the action descriptions and the goal. For convenience we list all the action descriptions in Table 2.

For a start, consider $A c t=\left\{\right.$ enter $_{i}$, leave $_{i}$, reveal $\left._{i}: i \in\{1,2\}\right\}$ where enter $_{i}$ and leave ${ }_{i}$ respectively have preconditions $\neg \mathrm{In}_{i}$ and $\ln _{i}$ and the obvious effects and where reveal ${ }_{i}$ requires to be in the room and has one unconditional effect ( $i$ knows the message) and one conditional effect: if the other agent is in the room then the message becomes common knowledge. We suppose, for simplicity, that there are no epistemic consequences when an agent enters or leaves the room because we are not interested in the epistemic status of 
$\ln _{1}$ and $\operatorname{In}_{2}$. Consider Goal $=\neg \ln _{1} \wedge \neg \ln _{2} \wedge S_{1} m \wedge S_{2} m$. The sequences

$$
\begin{aligned}
& \text { sol }_{1}=\left\langle\text { enter }_{1}, \text { reveal }_{1}, \text { leave }_{1}, \text { enter }_{2}, \text { reveal }_{2}, \text { leave }_{2}\right\rangle, \\
& \text { sol }_{2}=\left\langle\text { enter }_{1}, \text { enter }_{2}, \text { reveal }_{1}, \text { leave }_{1}, \text { leave }_{2}\right\rangle
\end{aligned}
$$

are both solutions of $\left\langle\mathrm{OA}\right.$, Act, $s_{0}$, Goal $\rangle$.

If we replace Goal by Goal' $=\neg \mathrm{I}_{1} \wedge \neg \mathrm{In}_{2} \wedge J S m$ then sol $_{2}$ still solves $\left\langle\mathrm{OA}\right.$, Act,$s_{0}$, Goal $\left.^{\prime}\right\rangle$, but sol $_{1}$ no longer does so.

Next, consider a variant of Act where the room is so small that it does not fit two persons: we have to replace enter ${ }_{i}$ by enter' $_{i}^{\prime}$ with precondition pre $\left(\right.$ enter $\left._{i}^{\prime}\right)=\neg \ln _{i} \wedge \neg \mathrm{In}_{j}$, for $i \neq j$. Let the resulting action description be $A c t^{\prime}$. While there is still a solution of $\left\langle\mathrm{OA}, A c t^{\prime}, s_{0}\right.$, Goal $\rangle$, there is no solution of $\left\langle\mathrm{OA}, A c t^{\prime}, s_{0}\right.$, Goal $\left.^{\prime}\right\rangle$.

Let us augment the set of actions by adding a further action informs $s_{1,2} m$ whose precondition is that both agents are in the same place, that the speaker 1 knows whether $m$ and that it is possible for her that $m$ is informative for the hearer 2. Let the resulting set of actions be $A c t^{\prime \prime}$. Then the sequence

$$
\text { sol }_{3}=\left\langle\text { enter }_{1}, \text { reveal }_{1}, \text { leave }_{1}, \text { informs }_{1,2} m\right\rangle
$$

becomes a further solution of $\left\langle\mathrm{OA}, A c t^{\prime \prime}, s_{0}\right.$, Goal $\rangle$ as well as of $\left\langle\mathrm{OA}, A c t^{\prime \prime}, s_{0}\right.$, Goal $\left.^{\prime}\right\rangle$. Observe that sol $_{3}$ is shorter than the other solutions.

Let us finally modify Act" into Act"' by a more demanding precondition of informing, namely by requiring that the speaker knows that her utterance is relevant for the hearer. Then $\mathrm{Sol}_{3}$ is no longer a solution of $\left\langle\mathrm{OA}, A c t^{\prime \prime}, s_{0}\right.$, Goal $\rangle$ nor of $\left\langle\mathrm{OA}, A c t^{\prime \prime}, s_{0}\right.$, Goal $\left.^{\prime}\right\rangle$ : the speaker lacks knowledge about the hearer. It becomes a solution again if we make the hypothesis that there is initial common knowledge of ignorance about $m$, i.e., if we set ${ }_{690} s_{0}^{\prime}=\left\{m, J S S_{1} m, J S S_{2} m\right\}$.

\subsection{Selective communication}

The following example is from Kominis and Geffner: 


\begin{tabular}{|l|l|l|}
\hline action & precondition & conditional effects \\
\hline goright $_{a}$ & $\mathrm{~T}$ & $\left\{\left\langle p_{a, k},\left\{p_{a, k+1}\right\},\left\{p_{a, k}\right\}\right\rangle: 1 \leq k \leq 3\right\}$ \\
goleft $_{a}$ & $\mathrm{~T}$ & $\left\{\left\langle p_{a, k},\left\{p_{a, k-1}\right\},\left\{p_{a, k}\right\}\right\rangle: 2 \leq k \leq 4\right\}$ \\
sense $_{a, q}$ & $p_{a, 2}$ & $\left\{\left\langle\mathrm{~T},\left\{S_{a} q\right\}, \emptyset\right\rangle\right\}$ \\
tell $_{a, q}$ & $S_{a} q$ & $\left\{\left\langle\varphi_{b}, S_{b} q, \emptyset\right\rangle,\left\langle\varphi_{c}, S_{c} q, \emptyset\right\rangle\right\}$ \\
\hline
\end{tabular}

Table 3: Action descriptions for the selective communication task

Let $a, b$, and $c$ be three agents in a corridor of four rooms ( $p_{1}, p_{2}, p_{3}$ and $p_{4}$ from left to right). The agents can move from a room to a contiguous room, and when agent $i$ communicates (tells) some information, all the agents that are in the same room or in a contiguous room, will hear what was communicated. For example, if agent $i$ expresses in $p_{3}$ his knowledge about $q$, all agents in rooms $p_{2}, p_{3}$ and $p_{4}$ will come to know it. We consider the problem where agent $a$ is initially in room $p_{1}, b$ in $p_{2}, c$ in $p_{3}$, and $a$ has to find out the truth value of a proposition $q$ and let $c$ know without agent $b$ learning it [53].

Let $p_{i, k}$ mean that agent $i$ is in room $p_{k}$, for $i \in\{a, b, c\}$ and $1 \leq k \leq 4$. Then the formula $\varphi_{b}=\bigvee_{1 \leq k, \ell \leq 4,|k-\ell| \leq 1}\left(p_{b, k} \wedge p_{b, \ell}\right)$ expresses that $b$ is close to $a$, and likewise for $\varphi_{c}$. We model this as a simple epistemic planning

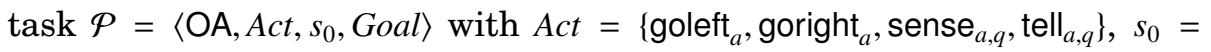
$\left\{p_{a, 1}, p_{b, 2}, p_{c, 3}\right\}$, Goal $=S_{c} q \wedge \neg S_{b} q 4$ The descriptions of the actions in Act are listed in Table 3 . Then the sequence

$$
\left\langle\text { goleft }_{a} \text {, } \text {,ense }_{a, q}, \text { goright }_{a}, \text { goright }_{a}, \text { goright }_{a}, \text { tel }_{a, q}\right\rangle
$$

is a solution of $\mathcal{P}$.

\footnotetext{
${ }^{4}$ Kominis and Geffner suppose that the possible initial states are common knowledge. We could express this by adding to $s_{0}$ the sets $\left\{J S S_{i} p_{a, 1}: i \in\{a, b, c\}\right\},\left\{J S S_{i} p_{b, 2}: i \in\{a, b, c\}\right\}$, and $\left\{J S S_{i} p_{c, 3}: i \in\{a, b, c\}\right\}$. We however disregard these hypotheses because our approach does not require it.
} 


\subsection{Pedestrian crossing}

In this 2-agent coordination problem that is derived from Lewis's [54], a pedestrian wants to cross a road at the same time as a car wants to reach its destination. The driver can either continue or stop to let the pedestrian cross. In the latter case the driver signals to the pedestrian, for example by flashing the car's headlights, that it is safe for the pedestrian to cross. We assume that the intentions of the pedestrian are obvious to the driver while the car is approaching (but not after it has driven away since the driver will not be looking in the rear-view mirror), but the pedestrian does not know this. Thus, before the pedestrian crosses the road, common knowledge must be established between the two agents (pedestrian and driver) of the pedestrian's intention to cross.

We can model this problem using the following propositional variables: $d_{c}$ is true if the car is driving forward, $r_{c}$ is true if the car has reached its destination; $r_{p}$ is true if the pedestrian has reached the other side of the road, $w r_{p}$ is true if the pedestrian wants to reach the other side of the road.

Then the planning task can be described as follows. The initial state is $s_{0}=\left\{d_{c}, w r_{p}, S_{c} w r_{p}\right\}$, the goal is Goal $=r_{p} \wedge r_{c}$, and the set of actions Act has four elements, three concerning the car and one the pedestrian, whose preconditions and effects are listed in Table 4. The action drivesOn ${ }_{c}$ has a precondition $d_{c} \wedge \neg r_{c}$ and (unique) conditional effect that the car reaches its destination, stops driving and no longer sees the value of $w r_{p}$ (whether the pedestrian wants to cross or not). Note that the fact that $S_{c} w r_{p}$ becomes false implies that $J S w r_{p}$ becomes false because the latter is an atomic (introspective) cause of the former, i.e., because $J S w r_{p} \in\left(S_{c} w r_{p}\right)^{\models}$. The action stopsAndSignals $s_{c}$ has a precondition $d_{c} \wedge w r_{p} \wedge S_{c} w r_{p}$ and (unique) conditional effect that the car stops and signals (by flashing its lights) that the driver knows that the pedestrian wants to cross which leads immediately to common knowledge of $w r_{p}$. The third possible action of the car is to restart (after a stop). Finally, the only action of the pedestrian is crosses $p$. 


\begin{tabular}{|l|l|l|}
\hline action & precondition & conditional effects \\
\hline drivesOn $_{c}$ & $d_{c} \wedge \neg r_{c}$ & $\left\{\left\langle\mathrm{~T},\left\{r_{c}\right\},\left\{d_{c}, S_{c} w r_{p}\right\}\right\rangle\right\}$ \\
stopsAndSignals $_{c}$ & $d_{c} \wedge w r_{p} \wedge S_{c} w r_{p}$ & $\left\{\left\langle\mathrm{~T},\left\{J S w r_{p}\right\},\left\{d_{c}\right\}\right\rangle\right\}$ \\
restarts $_{c}$ & $\neg d_{c} \wedge r_{p}$ & $\left\{\left\langle\mathrm{~T}, d_{c}, \emptyset\right\rangle\right\}$ \\
crosses $_{p}$ & $w r_{p} \wedge\left(\left(\neg d_{c} \wedge J S w r_{p}\right) \vee r_{c}\right)$ & $\left\{\left\langle\mathrm{T},\left\{r_{p}\right\},\left\{w r_{p}\right\}\right\rangle\right\}$ \\
\hline
\end{tabular}

Table 4: Action descriptions for the pedestrian crossing task

Then the two sequences of actions

$$
\begin{aligned}
& \left\langle\text { drivesOn }_{c}, \text { crosses }_{p}\right\rangle, \\
& \left\langle\text { stopsAndSignals }_{c}, \text { crosses }_{p}, \text { restarts }_{c}, \text { drivesOn }_{c}\right\rangle
\end{aligned}
$$

both solve our simple epistemic planning task $\left\langle\mathrm{OA}\right.$, Act, $s_{0}$, Goal $\rangle$.

\subsection{Authorisation via plenary meetings}

Here is a further example of actions with common knowledge preconditions. Consider a planning task involving cooperation between different agents that can be divided into $m$ different stages with tasks to be performed at each stage by each agent. Agents are only authorised to start stage $k+1$ if all tasks of stage $k$ have been completed and all agents have common knowledge of this. The only way this can be achieved is by having a plenary meeting at the end of each stage during which each agent announces that her stage- $k$ task has been completed 5

Each agent $i$ requires an authorisation $a_{i k}$ to start her stage- $k$ task. Initially, $a_{i 1}$ is true for all agents $i$, i.e., $s_{0}=\left\{a_{i 1}: i \in \mathbb{A}\right\}$. Let $t_{i k}$ represent

\footnotetext{
${ }^{5}$ The need for common knowledge can be motivated in the following manner: in order to go back to work, agents must know that other agents have completed their stage- $k$ task. Therefore agents need an acknowledgement of their announcing that they have completed this task in order to know that they are not preventing anybody else from going on with their work. But just like they need an acknowledgement, others need an acknowledgement of their acknowledgement, and so on, just like in the Byzantine Generals' Problem.
} 


\begin{tabular}{|l|l|l|}
\hline action & precondition & conditional effects \\
\hline doTask $_{\mathrm{ik}}$ & $a_{i k}$ & $\left\{\left\langle\mathrm{~T}, t_{i k} \wedge S_{i} t_{i k}, \emptyset\right\rangle\right\}$ \\
authorise $_{k}$ & $\bigwedge_{i \in \mathbb{A}} J S t_{i, k-1}$ & $\left\{\left\langle\mathrm{~T},\left\{a_{i k}: i \in \mathbb{A}\right\},\left\{p_{i}: i \in \mathbb{A}\right\}\right\rangle\right\}$ \\
goMeeting $_{\mathrm{i}}$ & $\mathrm{T}$ & $\left\{\left\langle\mathrm{T}, p_{i}, \emptyset\right\rangle\right\}$ \\
announce $_{\mathrm{i}}$ & $\bigwedge_{k \in \mathbb{A}} p_{k}$ & $\left\{\left\langle t_{i k} \wedge S_{i} t_{i k}, J S t_{i k}, \emptyset\right\rangle\right\}$ \\
\hline
\end{tabular}

Table 5: Action descriptions for the authorisation via plenary meetings task

the fact that agent $i \in \mathbb{A}$ has completed her stage- $k$ task. The propositional variable $p_{i}$ is true if agent $i$ is present at the meeting.

The preconditions and effects of the four action types doTask $\mathrm{ik}_{\mathrm{ik}}$, authorise $\mathrm{a}_{k}$, goMeeting $_{i}$, and announce ${ }_{i}$ are described in Table 5. For the action doTask ${ }_{i k}$, when an agent completes a task she sees this, but the other agents don't. The action authorise $e_{k}(k=2, \ldots, m)$ authorises the start of stage $k$. Authorisations for stage $k>1$ are only issued if there is common knowledge that all tasks of stage $k-1$ have been completed. They are thus issued at the end of the plenary meeting. A side effect of this is that all agents leave the

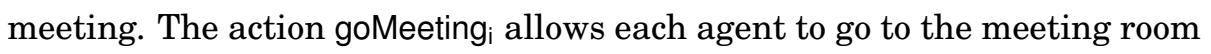
whenever she wants. Once everybody is present, each agent $i$ can announce that her task has been completed via the action announce ${ }_{\text {. }}$

The goal is common knowledge that all tasks have been completed:

$$
\text { Goal }=\bigwedge_{i \in \mathbb{A}} \bigwedge_{k=1, \ldots, m}\left(t_{i k} \wedge J S t_{i k}\right)
$$

With 2 agents and 2 stages, the following sequence is a solution:

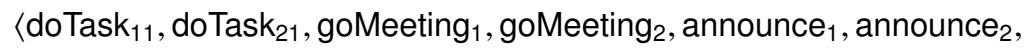
authorise 2 , doTask ${ }_{12}$, doTask 22 , goMeeting ${ }_{1}$, goMeeting 2 , announce ${ }_{1}$, announce 2 .

\subsection{The generalised gossip problem}

Let the set of agents be $\mathbb{A}=\{1, \ldots, n\}$ and let the set of propositional variables be $\mathbb{P}=\left\{s_{i}: i \in \mathbb{A}\right\}$. Let us describe the planning task for the 
generalised gossip problem of depth $k$ as a simple epistemic planning task $G_{k}=\left\langle\mathrm{OA}, A c t^{G_{k}}, s_{0}^{G_{k}}, G o a l^{G_{k}}\right\rangle$. The initial state is $s_{0}^{G_{k}}=\left\{S_{i} s_{i}: i \in \mathbb{A}\right\} \cup S$ for some $S \subseteq\left\{s_{i}: i \in \mathbb{A}\right\}$ (cf. Example 3). As we have seen in Example 4, the goal of obtaining shared knowledge of depth $k$ can be described in EL-O by

$$
\text { Goal }^{G_{k}}=\bigwedge_{i \in \mathbb{A}} \bigwedge_{\sigma \in \mathrm{OBS}^{+}, \ell(\sigma) \leq k} \sigma s_{i} .
$$

The set of actions is $A c t^{G_{k}}=\left\{\right.$ call $\left._{j}^{i}: i, j \in \mathbb{A}, i \neq j\right\}$, where pre $\left(\right.$ call $\left._{j}^{i}\right)=\mathrm{T}$ and where for every $0 \leq m<k$, every $\sigma_{m} \in \mathrm{OBS}^{\leq m}$ of length at most $m$ and every $r \in \mathbb{A}$ there are conditional effects $c e \in e f f\left(\right.$ call $\left._{j}^{i}\right)$ of the form:

$$
\begin{aligned}
\operatorname{cnd}(c e) & =S_{i} \sigma_{m} s_{r} \vee S_{j} \sigma_{m} s_{r} ; \\
\operatorname{ceff}^{+}(c e) & =\left\{\sigma S_{i} \sigma_{m} s_{r}: \sigma \in\left\{S_{i}, S_{j}\right\}^{\leq k-m-1}\right\} \cup\left\{\sigma S_{j} \sigma_{m} s_{r}: \sigma \in\left\{S_{i}, S_{j}\right\}^{\leq k-m-1}\right\} \\
& =\left\{\sigma \sigma_{m} s_{r}: \sigma \in\left\{S_{i}, S_{j}\right\}^{\leq k-m}\right\} ; \\
\operatorname{ceff}^{-}(c e) & =\emptyset .
\end{aligned}
$$

Remember that $\left\{S_{i}, S_{j}\right\}^{\leq k-m}$ denotes the set all sequences of observability operators $S_{i}$ and $S_{j}$ of length at most $k-m$. For $k=1$ we obtain $\operatorname{cnd}(c e)=$ $S_{i} s_{r} \vee S_{j} s_{r}, c e f f^{+}(c e)=\left\{S_{i} s_{r}, S_{j} s_{r}\right\}$, and ceff $f^{-}(c e)=\emptyset$, matching Example 7 .

We recall that the original gossip problem with $n \geq 4$ agents can be solved in $2(n-2)$ calls [46, 47, 48] and that there is no shorter solution. It is known that the generalised gossip problem $G_{k}$ can be solved in at most $(k+1)(n-2)$ calls [36] and that there is no shorter solution [4]. For instance, for $k=2$ and $n=5$ the sequence

$$
\text { call }_{3}^{1} \text {, call }{ }_{4}^{1} \text {, call }{ }_{5}^{2} \text {, call }{ }_{5}^{1} \text {, call }{ }_{3}^{1} \text {, call }{ }_{4}^{2}, \text { call }_{4}^{1} \text {, call }{ }_{5}^{1} \text {, call }{ }_{3}^{2}
$$

770 is a solution with $3 \times 3=9$ calls, which is therefore optimal.

\subsection{Generalised gossiping with ignorance goals}

We can also easily model ignorance goals. Given a set of atoms $A$, let $G-n e g_{k, A}=\left\langle\mathrm{OA}, A c t^{G-n e g_{k, A}}, s_{0}^{G-n e g_{k, A}}\right.$, Goal $\left.^{G-n e g_{k, A}}\right\rangle$ be the planning task for the generalised gossip problem of depth $k$ with the atoms of $A$ as the only negative 
goals. The initial state and the actions remain the same: $s_{0}^{G-n e g_{k, A}}=\left\{S_{i} s_{i}: i \in\right.$ $\mathbb{A}\} \cup S$ for some $S \subseteq\left\{s_{i}: i \in \mathbb{A}\right\}$ and Act $^{G-n \text { eg }_{k, A}}=\left\{\right.$ call $\left._{j}^{i}: i, j \in \mathbb{A}, i \neq j\right\}$; but the goal changes:

$$
\text { Goal }^{G-n e g_{k, A}}=\left(\bigwedge_{\sigma \in\left\{S_{i}: i \in \mathbb{A}\right\} \leq k} \bigwedge_{j \in \mathbb{A}, \sigma s_{j} \notin A} \sigma s_{j}\right) \wedge\left(\bigwedge_{\alpha \in A} \neg \alpha\right) .
$$

Here are some examples:

- $G$-neg $g_{1,\left\{S_{1} s_{2}\right\}}$ corresponds to the case where we want everyone to know all secrets, except that 1 should not know the secret of 2 ;

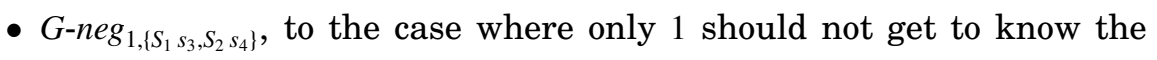
secret of 3 and 2, the secret of 4 ;

- $G$-neg $g_{2,\left\{S_{1} S_{2} s_{3}\right\}}$, to the case of epistemic depth 2 where only 1 should not know whether 2 knows the secret of 3 , while 1 and 2 should know the secret of 3 ;

- $G$-neg $g_{3,\left\{S_{1} S_{2} s_{3}\right\}}$ is the same as $G-n e g_{2,\left\{S_{1} S_{2} s_{3}\right\}}$ but with depth 3.

It was shown in [55] that the existence of a solution for gossiping with ignorance goals is an NP-complete problem.

\section{Encoding into PDDL and experiments}

In this section we report about some experiments with those simple epistemic planning tasks of Section 6 that are parametrised: general gossiping, general gossiping with ignorance goals, and authorisation via plenary meetings.

The experiments were done using three planners of the optimal track of IPC 2018: Planning-PDBs, Complementary1 and Complementary2. They all gave similar results for the two problems described below, so we chose to only show those for Planning-PDBs. The results were obtained on a GNU/Linux machine running on a 3,6 to $4,4 \mathrm{GHz}$ processor (Ryzen $73700 \mathrm{x}$ ) with $32 \mathrm{~GB}$ of RAM and a 30 minutes time limit. 
We first show how to encode simple planning tasks into the Planning

Fortunately almost all planners from the 2018 International Planning Competition (IPC 2018 $\sqrt{6}$ handle conditional effects and negative preconditions, and most of them handle disjunctive preconditions.

Given a formula $\varphi$ without introspective atoms, we define a recursive function $\operatorname{tr}_{P D D L}(\varphi)$ which returns the encoding of $\varphi$ into PDDL:

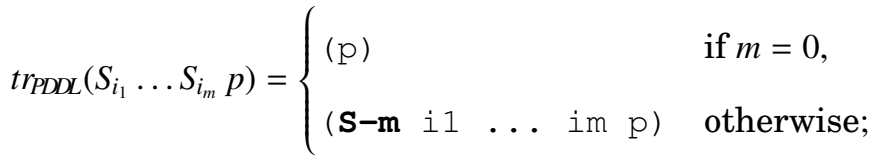

$$
\begin{aligned}
& \operatorname{tr}_{\text {PDDL }}\left(J S S_{i_{1}} \ldots S_{i_{m}} p\right)= \begin{cases}(\mathrm{JS} \mathrm{p}) & \text { if } m=0, \\
(\mathrm{JS}-\mathrm{m} \text { i } 1 \ldots \text { im p) } & \text { otherwise; }\end{cases} \\
& \operatorname{tr}_{P D D L}(\neg \varphi)=\left(\operatorname{not} \operatorname{tr}_{P D D L}(\varphi)\right) ; \\
& \operatorname{tr}_{P D D L}\left(\varphi_{1} \wedge \varphi_{2}\right)=\left(\text { and } \operatorname{tr}_{P D D L}\left(\varphi_{1}\right) \operatorname{tr}_{P D D L}\left(\varphi_{2}\right)\right) \text {. }
\end{aligned}
$$

with $p \in \mathbb{P}, m \geq 0$, and $i_{1}, \ldots, i_{m} \in \mathbb{A}$. In words, observability atoms $S_{i_{1}} \ldots S_{i_{m}} p$ and $J S S_{i_{1}} \ldots S_{i_{m}} p$ are respectively encoded by the special fluents S-m and 
JS, both with $m+1$ parameters. For $m=0$, the propositional variable $p$ is encoded as a fluent without parameters and $J S p$ is encoded as a special fluent (JS p). The initial state $s_{0}^{\Rightarrow} \cap \operatorname{Voc}(\mathcal{P})$ of $\operatorname{Exp}(\mathcal{P})$ is trivially encoded as a set of fluents thanks to $\operatorname{tr}_{P D D L}(\alpha)$. The formula Goal and the preconditions of every action can be encoded using $\operatorname{tr}_{P D D L}(\varphi)$ since they are all EL-O formulas. We note that we give no encoding into PDDL for atoms of the form $\sigma J S \alpha$ where $\sigma \in \mathrm{OBS}^{+}:$these atoms are introspective, and as the translation of planning tasks is normalised so as to remove any introspective atoms, we do not need to worry about encoding such atoms.

As we consider actions with conditional effects, the requirement flag :conditional-effects must be set.

Consider an action $\mathrm{a} \in$ Act. For every $c e \in$ eff (a) with $c e f f f^{+}(c e) \Rightarrow \cap \operatorname{Voc}(\mathcal{P})=$ $\left\{\alpha_{1}, \ldots, \alpha_{m}\right\}$ and $c$ eff ${ }^{-}(c e)^{\Leftarrow} \cap \operatorname{Voc}(\mathcal{P})=\left\{\beta_{1}, \ldots, \beta_{\ell}\right\}$, we add the conditional effect:

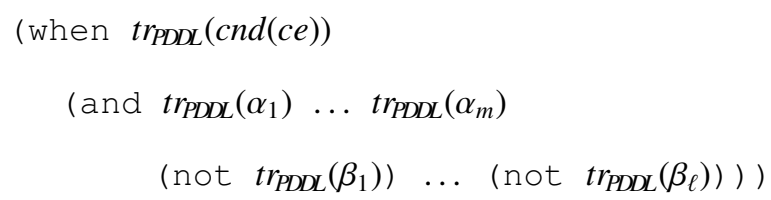

Example 10 (Example 7, ctd.). For the original gossip problem, the action description call ${ }_{2}^{1}$ of Example 7 is coded in PDDL as follows:

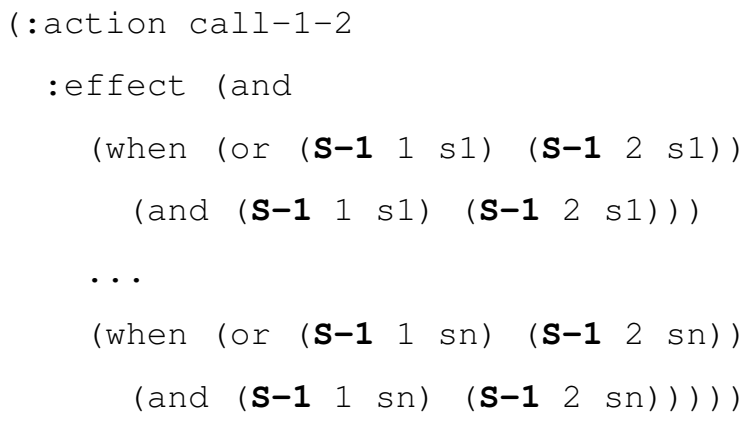

This is a direct encoding of a call into PDDL. Observe that we can generalise it to any $i$ and $j$ by writing:

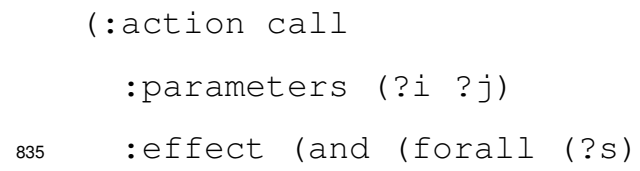




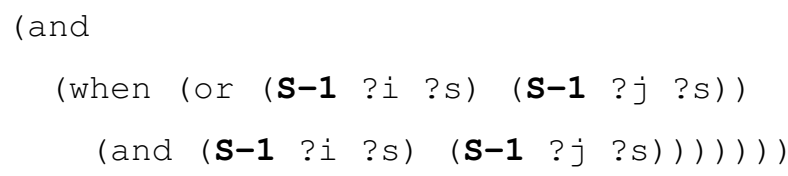

Finally, let us consider the generalised gossip problem of depth $k$ for the case of two agents 1 and 2. According to Section 6.6. call ${ }_{2}^{1}$ can be modelled as follows:

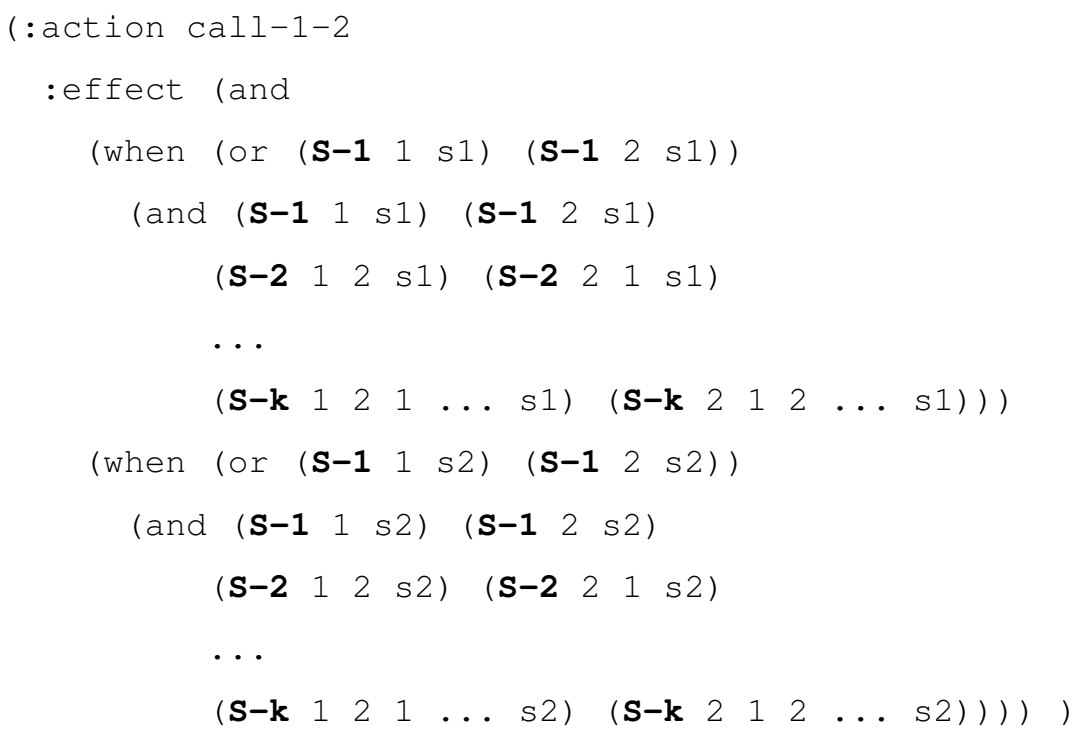

\subsection{Experiments}

In previous experiments we had used the planner FDSS-2014 [57] that handles conditional effects and negative and disjunctive preconditions for the gossip problem [10]. In more recent experiments we have used PlanningPDBs [58], as reported in the sequel. In comparison, the behaviour on the gossip problem was very similar, which is explained by the fact that in both cases the bottleneck was the grounding phase.

The resources available online 7 also contain an open-source PDDL generator for the gossip problem that was developed in Python 8 It allowed

7http://www.irit.fr/\%7EAndreas.Herzig/P/Ecai16.html

$\varepsilon$ https://github.com/FaustineMaffre/GossipProblem-PDDL-generator 
us to create the domain and problem files for the generalised gossip problem and its variant with ignorance goals. In its basic settings, the program needs the depth $k$ and the number of agents $n$. It is also possible to specify sets of negative goals, either directly by giving the index of agents such as $\left\{S_{1} s_{3}, S_{2} s_{4}\right\}$, or with constraints such as $\left\{S_{i} s_{j}: i \neq j\right.$ and $\left.j<3\right\}$ if we want no agent except 1 and 2 to know all secrets. Feeding the resulting files into a PDDL planner returns a solution to the corresponding gossip problem, namely a sequence of calls.

Generalised gossip problem. We ran FDSS-2014 and Planning-PDBs fixing a number of agents $n$ and increasing the depth $k$ until the first $k$ that leads to a timeout. We recall that the original gossip problem with $n \geq 4$ agents can be solved in $2 n-4$ calls and the generalised gossip problem (without negative goals) in at most $(k+1)(n-2)$ calls. For $k=2$ and $n=5$ the optimal plan found by both FDSS-2014 and Planning-PDBs was the following with 9 calls:

$$
\left\langle\text { call }_{2}^{1}, \text { call }_{3}^{1}, \text { call }_{5}^{4}, \text { call }_{4}^{1} \text {, call }{ }_{5}^{3} \text {, call }{ }_{5}^{2} \text {, call }{ }_{5}^{1}, \text { call }_{4}^{2}, \text { call }_{4}^{3}\right\rangle \text {. }
$$

More generally, for $k=2$ and $n \leq 5$, FDSS-2014 was able to find all optimal solutions that are computed by the protocol given in [36] and produced a timeout for $n=6$. For $n \geq 8$, FDSS-2014 produced a timeout already for $k=1$. The performance of Planning-PDBs was only slightly better: for $k=1$ the timeout occurred for $n=9$ and a solution was found for $n=8$ in $848 \mathrm{~s}$. Figure 1 gives the execution time of Planning-PDBs parametrised by the depth, while Figure 2 gives the execution time parametrised by the number of agents.

Generalised gossiping with ignorance goals. We ran FDSS-2014 and Planning-PDBs in the same manner as the generalised gossip problem, fixing a number of agents $n$ and increasing the depth $k$ until the first $k$ leading to a timeout. This time the timeout occurred for exactly the same $n$ and $k$. Table 6 contains the optimal plans that were produced for $n=4$ agents. The timeout occurred for $k=3$. 


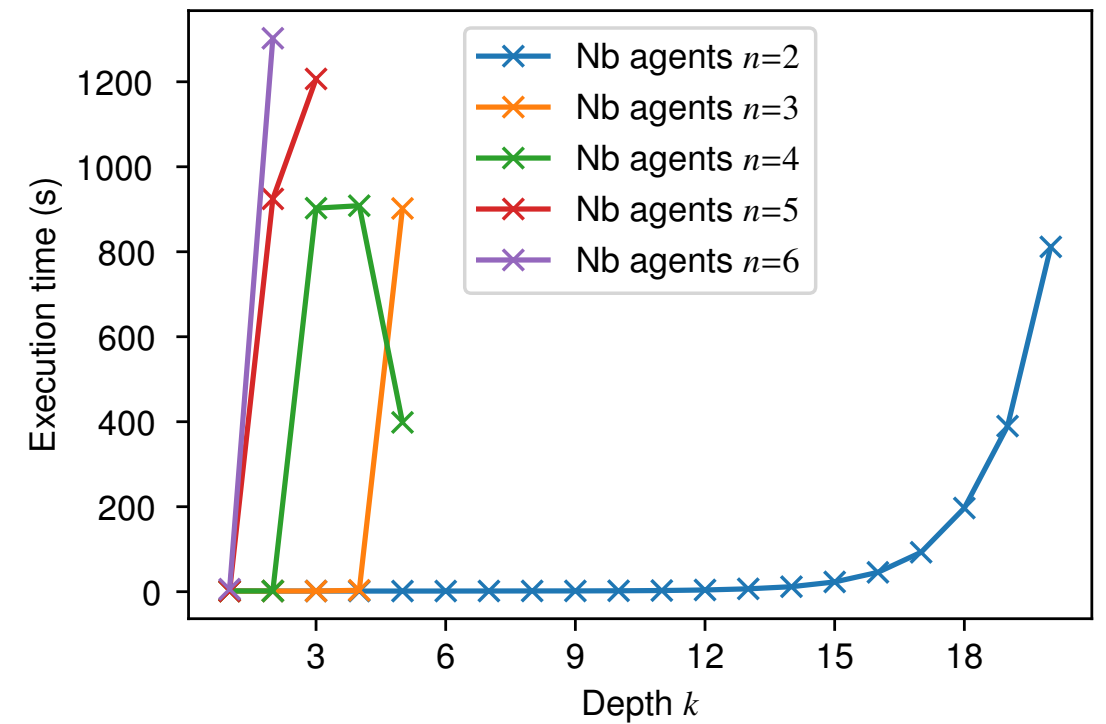

Figure 1: Generalised gossiping: time to find an optimal plan vs. epistemic depth (for different fixed numbers of agents)

\begin{tabular}{|l|l|}
\hline$G-$ neg $_{1,\left\{S_{1} s_{2}\right\}}$ & $\left\langle\right.$ call $_{3}^{1}$, call $_{4}^{1}$, call $_{4}^{2}$, call $\left._{4}^{3}\right\rangle$ \\
$G-$ neg $_{1,\left\{S_{1} s_{3}, S_{2} s_{4}\right\}}$ & $\left\langle\right.$ call $_{2}^{1}$, call $_{4}^{1}$, call $_{3}^{2}$, call $\left._{4}^{3}\right\rangle$ \\
$G-n e g_{2,\left\{S_{1} S_{2} s_{3}\right\}}$ & $\left\langle\right.$ call $_{2}^{1}$, call \\
4 \\
G- call
\end{tabular}

Table 6: Generalised gossiping with ignorance goals: optimal plans found by FDSS-2014 and Planning-PDBs, for $n=4$ agents 


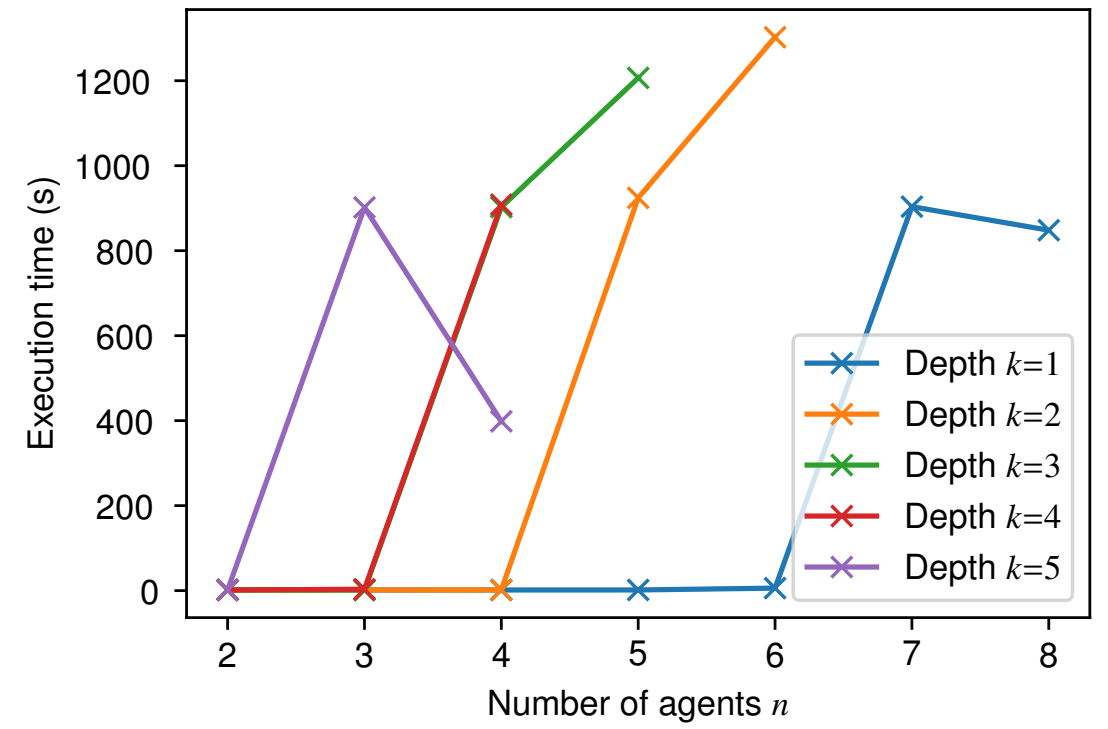

Figure 2: Generalised gossiping: time to find an optimal plan vs. number of agents (for different fixed epistemic depths)

Authorisation via plenary meetings. We ran Planning-PDBs on the plenary meetings problem for varying numbers of agents $n$ and numbers of meetings ('steps') $m$.

Figure 3 shows the time needed by Planning-PDBs to find an optimal plan, in seconds, relative to the number of agents, where each curve plotted is for a fixed number of meetings. For $m=1$ the timeout obtained when $n=7$; for $m=2$ when $n=5$; and for $m=3$ when $n=4$.

Figure 4 depicts the time to find an optimal plan w.r.t. the number of meetings, each curve being for a fixed number of agents. The curve for two agents provides some evidence for an exponential effect depending on the number of meetings. 


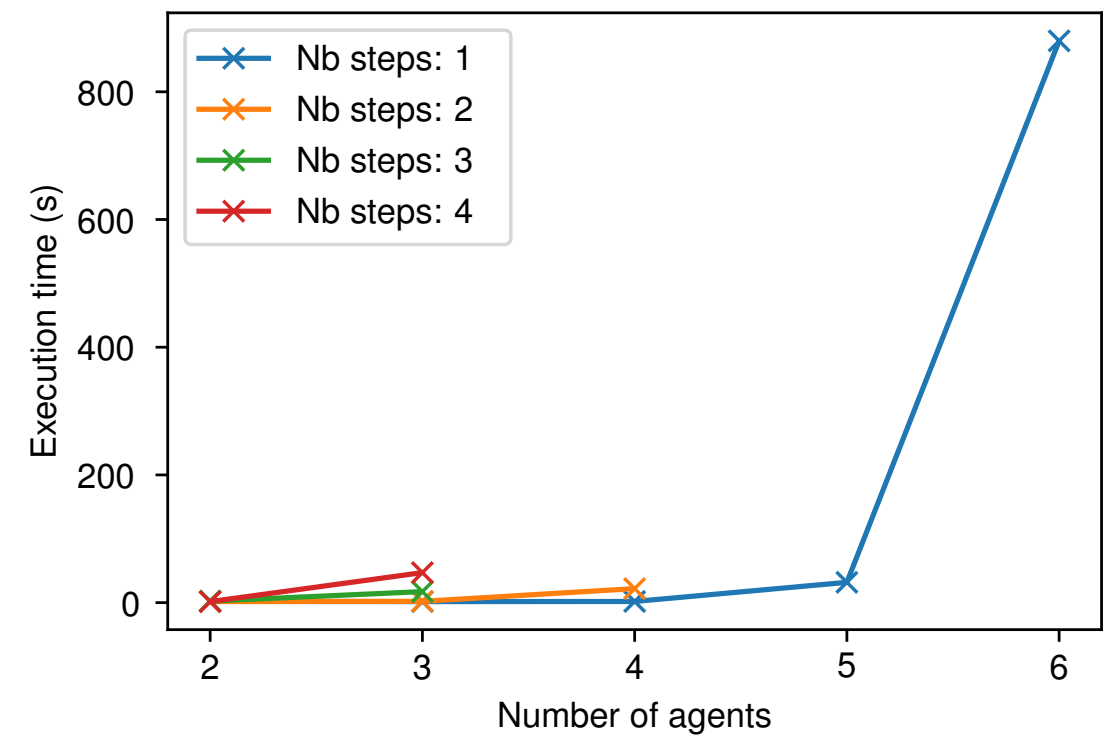

Figure 3: Plenary meetings: time to find an optimal parallel plan vs. number of agents (for different fixed numbers of meetings)

\section{Related work}

We now compare our approach with DEL-based planning as well as with the three existing approaches that can be viewed as particular cases of it. The latter either restrict the language to epistemic literals or restrict DEL's event models in a way such that actions are public or semi-private.

\subsection{Planning with DEL event models}

DEL-based planning was studied extensively in the literature in the last ten years [15, 16, 17, 18, 59, 19]. We show that our action descriptions can capture several important kinds of DEL event models (precisely, public, private and semi-private announcements) and the other way round, we show for some of our action descriptions how they correspond to DEL event models. One might expect that all such descriptions have a corresponding DEL event model; however, the correspondence is not obvious due to fundamen- 


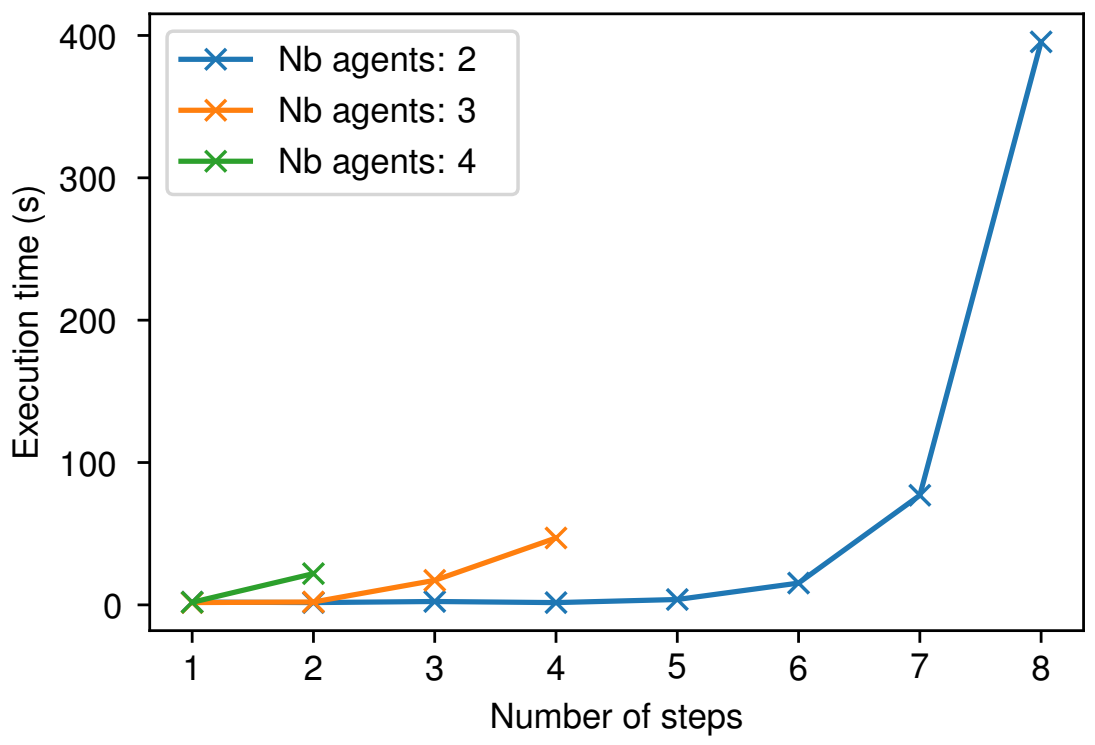

Figure 4: Plenary meetings: time to find an optimal parallel plan vs. number of meetings (for different fixed numbers of agents)

tal differences in the way epistemic effects are modelled. We explain this in detail in Section 8.1 .5 .

\subsubsection{Event models and product updates}

A DEL event model is a tuple Evt $=\left\langle W^{\mathrm{Evt}}, \sim_{\mathbb{A}}^{\mathrm{Evt}},\left\{\sim_{i}^{\mathrm{Evt}}\right\}_{i \in \mathbb{A}}, p r e^{\mathrm{Evt}}\right.$, post $\left.{ }^{\mathrm{Evt}}\right\rangle$ where $W^{\text {Evt }}$ is a finite set of events; $\sim_{\mathbb{A}}^{\text {Evt }}$ and the $\sim_{i}^{\text {Evt }}$ are equivalence relations on $W^{\text {Evt }}$ such that every $\sim_{i}^{\text {Evt }}$ is a subset of $\sim_{\mathbb{A}}^{\text {Evt }}$; pre $e^{\text {Evt }}: W^{\text {Evt }} \longrightarrow \mathcal{L}_{\mathrm{EL}}$ maps events to their preconditions; and post ${ }^{\mathrm{Evt}}: W^{\mathrm{Evt}} \longrightarrow\left(\mathbb{P} \longrightarrow \mathcal{L}_{\mathrm{EL}}\right)$ maps events to partial functions such that for every $e \in W^{\mathrm{Evt}}, \operatorname{post}^{\mathrm{Evt}}(e)$ is undefined almost everywhere. A pointed event model is a pair (Evt,e) where $e \in W^{\mathrm{Evt}}$ is the designated event (or the actual event). A multipointed event model is a pair (Evt, $E$ ) where $E \subseteq W^{\text {Evt }}$ is such that for any two distinct events $e, e^{\prime}$ in $E$, the preconditions of $e$ and $e^{\prime}$ are incompatible, that is, $p r e^{\mathrm{Evt}}(e) \wedge p r e^{\mathrm{Evt}}\left(e^{\prime}\right)$ is unsatisfiable.

Here are some examples and their representations as graphs, in which 
$\operatorname{Assign}(p, \varphi)$ :

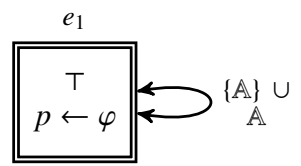

$\operatorname{PubAnn}(\varphi)$ :

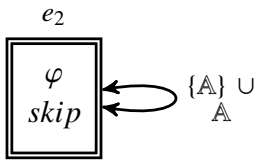

Figure 5: DEL event models for the public assignment of $\varphi$ to $p$ (on the left) and for the truthful public announcement of $\varphi$ (on the right).

nodes are events, labelled arrows represent the equivalence relations, the actual event is denoted via a double outline, and pre- and postconditions are given respectively in the top and bottom line in the nodes. An undefined postcondition is denoted by skip. We will often omit transitive and reflexive arrows for clarity.

1. The public assignment of $\varphi$ to $p$, i.e., the event where $p$ publicly gets the truth value of $\varphi$, is modelled by an event model Assign $(p, \varphi)$ with a single point $e_{1}$ whose precondition is $\mathrm{T}$, with total relations, i.e., such that $\sim_{\mathbb{A}}^{\text {Evt }}=\sim_{i}^{\text {Evt }}=\left\{\left\langle e_{1}, e_{1}\right\rangle\right\}$, and with the postcondition function $\operatorname{post}^{\mathrm{Evt}}\left(e_{1}\right)$ such that post ${ }^{\mathrm{Evt}}\left(e_{1}\right)(p)=\varphi$ and undefined for all $q \neq p$. This event model is shown on the left-hand side in Figure 5 .

2. The truthful public announcement of a formula $\varphi$ is modelled by an event model PubAnn $(\varphi)$, represented on the right-hand side of Figure 5, with a single point $e_{2}$ whose precondition is $\varphi$, with total relations and with an undefined postcondition function.

3. The truthful semi-private announcement whether $\varphi$ to agent $i$ is when $i$ learns whether $p$ and the other agents only learn that $i$ learns whether $p$ without learning whether $p$ themselves. This is modelled by an event model SemiPrivAnn $(\varphi, i)$ with two points $e^{+}$and $e^{-}$where $p r e^{\text {Evt }}\left(e^{+}\right)=$ $\varphi$ and $\operatorname{pre}^{\mathrm{Evt}}\left(e^{-}\right)=\neg \varphi$, with an undefined postcondition function, and with $\sim_{\mathbb{A}}^{\text {Evt }}=\sim_{j}^{\text {Evt }}=\left\{e^{+}, e^{-}\right\} \times\left\{e^{+}, e^{-}\right\}$for every $j \neq i$ and $\sim_{i}^{\text {Evt }}=\left\{\left\langle e^{+}, e^{+}\right\rangle,\left\langle e^{-}, e^{-}\right\rangle\right\}$. When we want to model that $i$ learns that $\varphi$ then $e^{+}$is the designated event; otherwise it is $e^{-}$, as is the case in Figure 6 
$\operatorname{SemiPrivAnn}(\varphi, i)$ :

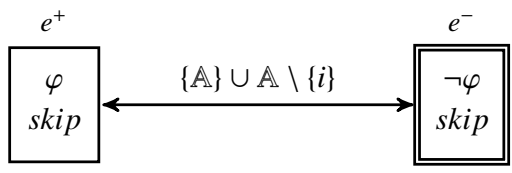

Figure 6: DEL event model for the truthful semi-private announcement of $\neg \varphi$ to agent $i$.

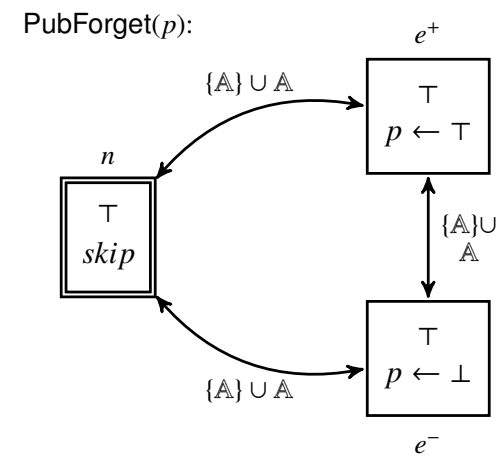

Figure 7: DEL event model for the public forgetting of $p$.

4. The public forgetting of a propositional variable $p$ by all agents [60] can be captured by an event model PubForget $(p)$ with three points $n$, $e^{+}$and $e^{-}$where $p r e^{\mathrm{Evt}}(n)=\operatorname{pr} e^{\mathrm{Evt}}\left(e^{+}\right)=\operatorname{pre} e^{\mathrm{Evt}}\left(e^{-}\right)=\mathrm{T}$, where $\sim_{\mathbb{A}}^{\text {Evt }}=$ $\sim_{i}^{\text {Evt }}=\left\{n, e^{+}, e^{-}\right\} \times\left\{n, e^{+}, e^{-}\right\}$for every $i$, and where post ${ }^{\text {Evt }}$ is such that $\operatorname{post}^{\mathrm{Evt}}(n)(p)=p$, post $^{\mathrm{Evt}}\left(e^{+}\right)(p)=\mathrm{\top}$, post $^{\mathrm{Evt}}\left(e^{-}\right)(p)=\perp$, and post ${ }^{\mathrm{Evt}}(n)=$ skip. This event model is represented in Figure 7. Note that a (singlepointed) event model with two points would not do the job: it would force commitment to some ontic event taking place.

Given a Kripke model $M=\left\langle W, \sim_{\mathbb{A}},\left\{\sim_{i}\right\}_{i \in \mathbb{A}}, V\right\rangle$ as defined in Section 4 and 955 an event model Evt, the product update of $M$ by Evt is the Kripke model $M \otimes \mathrm{Evt}=\left\langle W^{\prime}, \sim_{\mathbb{A}}^{\prime},\left\{\sim_{i}^{\prime}\right\}_{i \in \mathbb{A}}, V^{\prime}\right\rangle$ with

- $W^{\prime}=\left\{(s, e): s \in W, e \in W^{\mathrm{Evt}}\right.$, and $\left.(M, s) \vDash p r e^{\mathrm{Evt}}(e)\right\}$;

- $(s, e) \sim_{\mathbb{A}}^{\prime}(t, f)$ iff $s \sim_{\mathbb{A}} t$ and $e \sim_{\mathbb{A}}^{\text {Evt }} f$; 
- $(s, e) \sim_{i}^{\prime}(t, f)$ iff $s \sim_{i} t$ and $e \sim_{i}^{\text {Evt }} f$, for every $i \in \mathbb{A}$; formula obtained by exhaustively applying the reduction axioms of Section 4.4.) So $M^{\mathrm{EL}-\mathrm{O}}, s \vDash p r e^{\mathrm{Evt}}(e)$ exactly when $R_{\mathrm{a}(\mathrm{Evt}, e)}^{\mathrm{EL}, \mathrm{O}}$ is defined at $s$. For such $\left\{p: p o s t^{\text {Evt }}(e)(p)\right.$ is defined and $\left.(M, s) \vDash p o s t^{\text {Evt }}(e)(p)\right\}$.

The product update of a pointed $\operatorname{Kripke} \operatorname{model}(M, s)$ with a pointed event model (Evt, $e$ ) is the pointed Kripke model $(M \otimes \operatorname{Evt},(s, e))$, defined only when $(M, s) \vDash p r e^{\mathrm{Evt}}(e)$. The product update of a pointed model $(M, s)$ with a multipointed event model $(\mathrm{Evt}, E)$ is the pointed model $(M \otimes \operatorname{Evt},(s, e))$ where $e \in E$ is such that $(M, s) \vDash p r e^{\operatorname{Evt}}(e)$, defined when such an $e$ exists in $E$. In that case the hypothesis in the definition of a multipointed event model that the preconditions of different events are incompatible ensures that it is unique.

\subsubsection{Bisimulations}

We recall a standard notion in modal logics that will be instrumental to prove our results: a bisimulation [61] between two Kripke models $M=$ $\left\langle W, \sim_{\mathbb{A}},\left\{\sim_{i}\right\}_{i \in \mathbb{A}}, V\right\rangle$ and $M^{\prime}=\left\langle W^{\prime}, \sim_{\mathbb{A}}^{\prime},\left\{\sim_{i}^{\prime}\right\}_{i \in \mathbb{A}}, V^{\prime}\right\rangle$ is a relation $Z \subseteq W \times W^{\prime}$ such that for all $w \in W$ and $w^{\prime} \in W^{\prime}$ such that $w Z w^{\prime}: V(w)=V^{\prime}\left(w^{\prime}\right)$ (atomic); if $w \sim_{\mathbb{A}} u$ then $u Z u^{\prime}$ and $w^{\prime} \sim_{\mathbb{A}}^{\prime} u^{\prime}$ for some $u^{\prime}$, and if $w \sim_{i} u$ then $u Z u^{\prime}$ and

$975 w^{\prime} \sim_{i}^{\prime} u^{\prime}$ for some $u^{\prime}$ (forth); if $w^{\prime} \sim_{\mathbb{A}}^{\prime} u^{\prime}$ then $u Z u^{\prime}$ and $w \sim_{\mathbb{A}} u$ for some $u$, and if $w^{\prime} \sim_{i}^{\prime} u^{\prime}$ then $u Z u^{\prime}$ and $w \sim_{i} u$ for some $u$ (back). Two pointed models $(M, w)$ and $\left(M^{\prime}, w^{\prime}\right)$ are bisimilar if there exists a bisimulation $Z$ between $M$ and $M^{\prime}$ such that $w Z w^{\prime}$. In that case, we have $M, w \vDash \varphi$ iff $M^{\prime}, w^{\prime} \vDash \varphi$ for every formula $\varphi \in \mathcal{L}_{\text {EL }}$ [35].

\subsubsection{From DEL event models to EL-O action descriptions}

In what follows we show that we can express several kinds of event models, where we sometimes restrict preconditions to literals and conjunctions thereof. We are going to associate an EL-O action description a(Evt,e) to a given pointed event model (Evt, $e$ ). In all cases, the precondition of a(Evt,e) is $p r e^{\mathrm{Evt}}(e)$. (More precisely, it is the reduction of $p r e^{\mathrm{Evt}}(e)$ to an $\mathcal{L}_{\text {bool }}(\mathrm{OA})$

- $V^{\prime}((s, e))=\left\{p: p o s t^{\mathrm{Evt}}(e)(p)\right.$ is undefined and $\left.(M, s) \vDash p\right\} \cup$ 
points $s$, it can be shown that the pointed product update $\left(M^{\mathrm{EL}-\mathrm{O}} \otimes \mathrm{Evt},(s, e)\right)$ is bisimilar to the pointed Kripke model $\left(M^{\mathrm{EL}-\mathrm{O}}, t\right)$ for the state $t$ such that $s R_{\mathrm{a}(\mathrm{Evt}, e)}^{\mathrm{EL}-\mathrm{O}} t$.

First, let us look at some kinds of public assignments. Assign $(p, T)$ is captured by the action description $\operatorname{a}\left(\operatorname{Assign}(p, T), e_{1}\right)=\langle\top,\{\langle\top,\{J S p, p\}, \emptyset\rangle\}\rangle$ and $\operatorname{Assign}(p, \perp)$ is captured by $\mathrm{a}\left(\operatorname{Assign}(p, \perp), e_{1}\right)=\langle\top,\{\langle T,\{J S p\},\{p\}\rangle\}\rangle$. Finally, Assign $(p, \neg p)$ is the public toggling of the truth value of $p$ and is captured by an action description with two conditional effects

$$
\operatorname{a}\left(\operatorname{Assign}(p, \neg p), e_{1}\right)=\langle\top,\{\langle\neg p,\{p\}, \emptyset\rangle,\langle p, \emptyset,\{p\}\rangle\}\rangle .
$$

Observe that, contrarily to Assign $(p, T)$ and Assign $(p, \perp), \operatorname{Assign}(p, \neg p)$ does not modify $J S p$ : if an agent does not know whether $p$ before the public toggling of $p$ then she also does not know whether $p$ afterwards.

Second, let us look at some kinds of public announcements. The truthful public announcement of a propositional variable $p$ is captured by the action description with precondition $p$, unconditional positive effect $J S p$, and without negative effects: $\mathrm{a}\left(\operatorname{PubAnn}(p), e_{2}\right)=\langle p,\{\langle T,\{J S p\}, \emptyset\rangle\}\rangle$. Symmetrically, the public announcement of $\neg p$ is captured by a $\left(\operatorname{PubAnn}(\neg p), e_{2}\right)=$ $\langle\neg p,\{\langle T,\{J S p\}, \emptyset\rangle\}\rangle$. More generally, we can capture the public announcement of conjunctions of atoms and negations of atoms:

$\mathrm{a}\left(\operatorname{PubAnn}\left(\left(\bigwedge_{\alpha \in A^{+}} \alpha\right) \wedge\left(\bigwedge_{\alpha \in A^{-}} \neg \alpha\right)\right), e_{2}\right)=\left\langle\left(\bigwedge_{\alpha \in A^{+}} \alpha\right) \wedge\left(\bigwedge_{\alpha \in A^{-}} \neg \alpha\right),\left\{\left\langle\top,\left\{J S \alpha: \alpha \in A^{+} \cup A^{-}\right\}, \emptyset\right\rangle\right\}\right\rangle$.

The precondition guarantees that for example the public announcement of $J S p \wedge \neg S_{i} p$ cannot be made. Here are some examples of public announcements of the above conjunctions. We also add the equivalent formulation in the standard epistemic language.

$$
\begin{aligned}
\mathrm{a}\left(\operatorname{PubAnn}\left(\neg K_{i} p \wedge \neg K_{i} \neg p\right), e_{2}\right)=\mathrm{a}\left(\operatorname{PubAnn}\left(\neg S_{i} p\right), e_{2}\right) & =\left\langle\neg S_{i} p,\left\{\left\langle\top,\left\{J S S_{i} p\right\}, \emptyset\right\rangle\right\}\right\rangle, \\
\mathrm{a}\left(\operatorname{PubAnn}\left(K_{i} p\right), e_{2}\right)=\mathrm{a}\left(\operatorname{PubAnn}\left(p \wedge S_{i} p\right), e_{2}\right) & =\left\langle p \wedge S_{i} p,\{\langle\top,\{J S p\}, \emptyset\rangle\}\right\rangle, \\
\mathrm{a}\left(\operatorname{PubAnn}\left(p \wedge \neg K_{i} p\right), e_{2}\right)=\mathrm{a}\left(\operatorname{PubAnn}\left(p \wedge \neg S_{i} p\right), e_{2}\right) & =\left\langle p \wedge \neg S_{i} p,\{\langle\top,\{J S p\}, \emptyset\rangle\}\right\rangle .
\end{aligned}
$$

In the announcement of $p \wedge S_{i} p$ and of the Moore sentence $p \wedge \neg S_{i} p$ we have 
dropped $J S S_{i} p$ from the add-list because it already contains $J S p$ (cf. the EL-O axiom $V i s_{5}$.

Third, the semi-private announcement to $i$ that $p$ is true is captured by the action description

$$
\text { a(SemiPrivAnn } \left.(p, i), e^{+}\right)=\left\langle p,\left\{\left\langle\top,\left\{S_{i} p, J S S_{i} p\right\}, \emptyset\right\rangle\right\}\right\rangle .
$$

So the effect is that $i$ sees whether $p$ and that all agents jointly see that; in other words, that it becomes common knowledge that $i$ sees whether $p$.

Fourth, the event model PubForget $(p)$ of publicly forgetting $p$ corresponds to the action description

$$
\mathrm{a}(\operatorname{PubForget}(p), n)=\left\langle\mathrm{T},\left\{\left\langle\mathrm{T},\left\{J S S_{i} p: i \in \mathbb{A}\right\},\left\{S_{i} p: i \in \mathbb{A}\right\}\right\rangle\right\}\right\rangle .
$$

That is, all $S_{i} p$ become false and this becomes common knowledge.

Proposition 15. For each of the above event models Evt:

1. $M^{\mathrm{EL}-\mathrm{O}}, s \vDash p r e^{\mathrm{Evt}}(e)$ if and only if $R_{\mathrm{a}(\mathrm{Evt}, e)}^{\mathrm{EL}-\mathrm{O}}$ is defined at $s$;

2. If $s R_{\mathrm{a}(\mathrm{Ev}, e)}^{\mathrm{EL}, \mathrm{O}} s^{\prime}$ then $\left(M^{\mathrm{EL}-\mathrm{O}}, s^{\prime}\right)$ and $\left(M^{\mathrm{EL}-\mathrm{O}} \otimes \mathrm{Evt},(s, e)\right)$ are bisimilar.

Proof. The first item is the case because, as already mentioned, the precondition of the action descriptions $\mathrm{a}(\mathrm{Evt}, e)$ are all equivalent to $p r e^{\mathrm{Evt}}(e)$.

We sketch the proof of the second item for the public announcement of $p$. Let $M^{\mathrm{EL}-\mathrm{O}} \otimes \operatorname{PubAnn}(p)=\left\langle W, \sim_{\mathbb{A}},\left\{\sim_{i}\right\}_{i \in \mathbb{A}}, V\right\rangle$, and consider $s \in W^{\mathrm{EL}-\mathrm{O}}$ such that $s R_{\mathrm{a}(\operatorname{PubAnn}(p))}^{\mathrm{EL}} s^{\prime}$, i.e., such that $s^{\prime}=s \cup\{J S p\} \Rightarrow$. We show that the pointed models $\left(M^{\mathrm{EL}-\mathrm{O}}, s^{\prime}\right)$ and $\left(M^{\mathrm{EL}-\mathrm{O}} \otimes \operatorname{PubAnn}(p),\left(s, e_{2}\right)\right)$ are bisimilar, where $e_{2}$ is the single event of the event model PubAnn $(p)$. To that end we define the relation $Z$ between $W^{\mathrm{EL}-\mathrm{O}}$ and $W$ as: $s^{\prime} Z\left(s, e_{2}\right)$ iff $s^{\prime}=s \cup\{J S p\}^{\Rightarrow}$. It can be checked that the three conditions for $Z$ being a bisimulation are satisfied: atomic, forth, and back.

The other proofs are similar; we only sketch the case of public forgetting of $p$, PubForget $(p)$. Suppose $s R_{\mathrm{a}(\operatorname{PubForget}(p))}^{\mathrm{EL}} t$, i.e.,

$$
t=\left(s \backslash\left\{S_{i} p: i \in \mathbb{A}\right\}^{\models}\right) \cup\left\{J S S_{i} p: i \in \mathbb{A}\right\} \Rightarrow
$$

For every $s$, this state $t$ is unique: we denote it by $R_{\mathrm{a}(\operatorname{\mathrm {EL}} \mathrm{ObForget}(p))}(s)$. We show that $\left(M^{\mathrm{EL}-\mathrm{O}}, t\right)$ and $\left(M^{\mathrm{EL}-\mathrm{O}} \otimes \mathrm{PubForget}(p),(s, n)\right)$ are bisimilar via the following 
relation $Z$ between $W$ and $W^{\mathrm{EL}-\mathrm{O}}$ :

$$
\begin{aligned}
Z= & \left\{\left\langle R_{\mathrm{a}(\text { PubForget }(p))}^{\mathrm{EL}-\mathrm{O}}(s),(s, n)\right\rangle\right\} \cup \\
& \left\{\left\langle R_{\mathrm{a}(\text { PubForget }(p))}^{\mathrm{EL}-\mathrm{O}}(s) \cup\{p\},\left(s, e^{+}\right)\right\rangle\right\} \cup \\
& \left\{\left\langle R_{\mathrm{a}(\text { PubForget }(p))}^{\mathrm{EL}-\mathrm{O}}(s) \backslash\{p\},\left(s, e^{-}\right) .\right\rangle\right\}
\end{aligned}
$$

1025 It can be checked that $Z$ is indeed a bisimulation.

Something that cannot be modelled in our framework is the public announcement that $\neg K_{i} p$, or, expressed in the EL-O fragment, that $\neg\left(p \wedge S_{i} p\right)$. The reason is that it is not clear how the disjunctive effect of that announcement could be described in terms of an add-list.

\subsubsection{From EL-O action descriptions to DEL event models}

We now consider the converse direction: given an EL-O action description a, can we find a DEL event model (Evt, $E$ ) such that for any state $s \in$ $W^{\mathrm{EL}-\mathrm{O}}$, the product $\left(M^{\mathrm{EL}-\mathrm{O}} \otimes \mathrm{Evt},(s, e)\right)$ of $\left(M^{\mathrm{EL}-\mathrm{O}}, s\right)$ and $(\mathrm{Evt}, E)$ is defined iff $s \vDash \operatorname{pre}(\mathrm{a})$, and such that in that case, $\left(M^{\mathrm{EL}-\mathrm{O}} \otimes \mathrm{Evt},(s, e)\right)$ and $\left(M^{\mathrm{EL}-\mathrm{O}}, s^{\prime}\right)$ are bisimilar, where $s^{\prime}$ is the state such that $s R_{\mathrm{a}}^{\mathrm{EL}-\mathrm{O}} s^{\prime}$ ?

Let us here restrict our attention to a few very basic EL-O action descriptions in which there is a single conditional effect either adding or deleting one atom. The idea behind the construction of an equivalent event model is the following: when adding or deleting an atom from a world, only agents who see this atom know that it has been modified.

The easiest way to translate this into DEL event models is through the use of Bolander's edge-conditioned event models [62, 63]. Such models incorporate conditions on accessibility relations, so that a model is a tuple Evt $=\left\langle W^{\mathrm{Evt}}, R_{\mathbb{A}}^{\mathrm{Evt}},\left\{R_{i}^{\mathrm{Evt}}\right\}_{i \in \mathrm{A}}, p r e^{\mathrm{Evt}}\right.$, post $\left.{ }^{\mathrm{Evt}}\right\rangle$ where $R_{\mathbb{A}}^{\mathrm{Evt}}: W^{\mathrm{Evt}} \times W^{\mathrm{Evt}} \rightarrow \mathcal{L}_{\mathrm{EL}}$,

$R_{i}^{\text {Evt }}: W^{\text {Evt }} \times W^{\text {Evt }} \rightarrow \mathcal{L}_{\mathrm{EL}}$ for every $i \in \mathbb{A}$, and the rest is defined as previously. The product of such an edge-conditioned event model with a model $M=\left\langle W, \sim_{\mathbb{A}},\left\{\sim_{i}\right\}_{i \in \mathbb{A}}, V\right\rangle$ is $M \otimes$ Evt $=\left\langle W^{\prime}, \sim_{\mathbb{A}}^{\prime},\left\{\sim_{i}^{\prime}\right\}_{i \in \mathbb{A}}, V^{\prime}\right\rangle$ where

- $(s, e) \sim_{\mathbb{A}}^{\prime}(t, f)$ iff $s \sim_{\mathbb{A}} t$ and $(M, s) \models R_{\mathbb{A}}^{\text {Evt }}(e, f)$ and $(M, t) \vDash R_{\mathbb{A}}^{\text {Evt }}(e, f)$; 


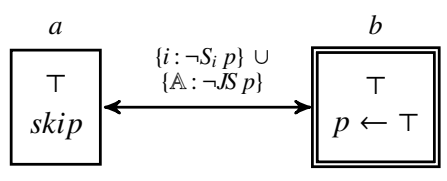

Figure 8: An event model equivalent to $\langle T,\{\langle T,\{p\}, \emptyset\rangle\}\rangle$.

- $(s, e) \sim_{i}^{\prime}(t, f)$ iff $s \sim_{i} t$ and and $(M, s) \vDash R_{i}^{\mathrm{Evt}}(e, f)$ and $(M, t) \vDash R_{i}^{\mathrm{Evt}}(e, f)$, for every $i \in \mathbb{A}$;

and the rest is defined as previously. As mentioned in [63], edge-conditioned event models can be simulated by standard event models, though the former are more succinct.

We now give a few examples of DEL event model translations of such simple action descriptions. We will only give the bisimulation relations without the proofs that they are indeed bisimulations, as those proofs are tedious but uncomplicated.

An equivalent event model to the assignment of $p$ to true, $\langle T,\{\langle T,\{p\}, \emptyset\rangle\}\rangle$, is given in Figure 8, The bisimulation relation $Z$ is as follows: for every state $s \in W^{\mathrm{EL}-\mathrm{O}}, s Z(s, a)$ and $(s \cup\{p\}) Z(s, b)$. If we wish to assign $p$ to false rather than true (action $\langle T,\{\langle T, \emptyset,\{p\}\rangle\}\rangle)$, we simply replace the assignment $p \leftarrow \top$ in the actual event by $p \leftarrow \perp$, and change the bisimulation relation so that $(s \backslash\{p\}) Z(s, b)$ for any $s \in W^{\mathrm{EL}-\mathrm{O}}$. This action can be interpreted as the value of $p$ (the property of an object) changing, while only agents who are looking at this object can see the change.

For the action $\left\langle\mathrm{T},\left\{\left\langle\mathrm{T},\left\{S_{i} \alpha\right\}, \emptyset\right\rangle\right\}\right\rangle$ making $S_{i} \alpha$ true, we need in the general case two actual events, one for when $\alpha$ is true and one for when it is false. An event model equivalent to this action description is given in Figure 9 . The corresponding bisimulation $Z$ is the following: for every state $s \in W^{\mathrm{EL}-\mathrm{O}}$,

- $\left(s \cup\left\{S_{i} \alpha\right\}\right) Z(s, b)$ if $s \vDash \alpha$;

- $\left(s \cup\left\{S_{i} \alpha\right\}\right) Z(s, c)$ if $s \vDash \neg \alpha$. 


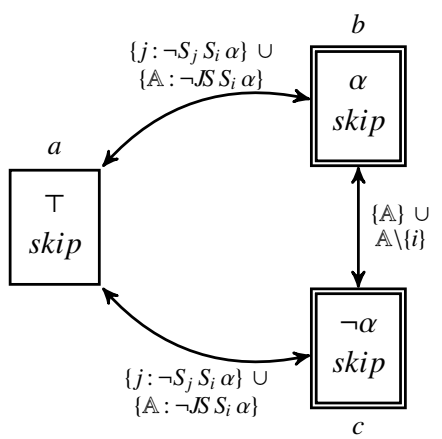

Figure 9: An event model equivalent to $\left\langle\mathrm{T},\left\{\left\langle\mathrm{T},\left\{S_{i} \alpha\right\}, \emptyset\right\rangle\right\}\right\rangle$.

A more complex example, given in Figure 10, is that of an event model equivalent to an action making the atom $S_{i} p$ false. This can be interpreted as agent $i$ looking away from $p$. Agents who saw agent $i$ looking at $p$ will see her look away, and the other agents will not be aware of this change. While this is a fairly natural action to consider, it is difficult to generalize to assigning $S_{i} \alpha$ to false for any given $\alpha$, as only propositional variables can receive assignments in $D E L$ event models. The bisimulation $Z$ in the case of Figure 10 is as follows: for every state $s \in W^{\mathrm{EL}-\mathrm{O}}$,

- $s Z(s, a)$;

- $\left(s \backslash\left\{J S p, S_{i} p\right\}\right) Z(s, b)$;

- $\left(s \backslash\left\{J S p, S_{i} p, p\right\}\right) Z(s, c)$ if $s \vDash p$;

- $\left((s \cup\{p\}) \backslash\left\{J S p, S_{i} p\right\}\right) Z(s, c)$ if $s \vDash \neg p$;

- $(s \backslash\{p\}) Z(s, d)$ if $s \vDash p$;

- $(s \cup\{p\}) Z(s, d)$ if $s \vDash \neg p$.

Let us give one more example, this time involving a precondition: private announcements. In an S5 setting, an announcement can only be private if no one is watching (as we cannot represent agents "believing that nothing has happened"). That is, we can only privately announce whether 


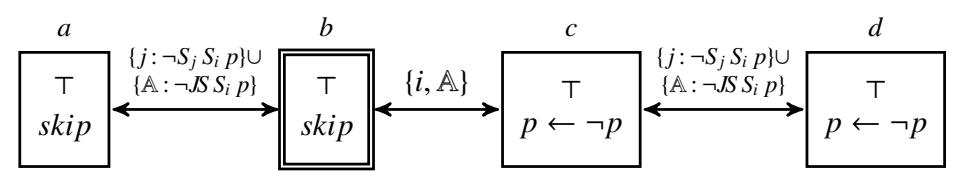

Figure 10: An event model equivalent to $\left\langle T,\left\{\left\langle\top, \emptyset,\left\{S_{i} p\right\}\right\rangle\right\}\right\rangle$.

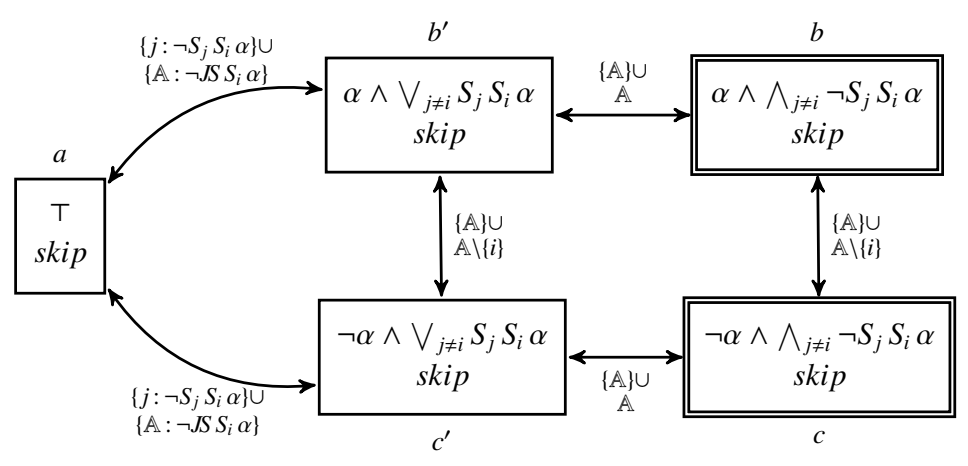

Figure 11: An event model equivalent to the private announcement whether $\alpha$ to $i$.

$\alpha$ is true to an agent $i$ if for all $j \neq i, \neg S_{j} S_{i} \alpha$ holds. The corresponding action description has condition $\bigwedge_{j \neq i} \neg S_{j} S_{i} \alpha$ and a single conditional effect $\left\langle\top,\left\{S_{i} \alpha\right\}, \emptyset\right\rangle$. The corresponding event model is given in Figure 11. The bisimulation $Z$ is: for every state $s \in W^{\mathrm{EL}-\mathrm{O}}, s Z(s, a)$, and $\left(s \cup S_{i} \alpha\right) Z(s, e)$ if $s \vDash p r e^{\text {Evt }}(e)$ for $e \in\left\{b, b^{\prime}, c, c^{\prime}\right\}$.

We stop here without delving into the territory of deleting longer atoms or combining several effects. It is, we hope, fairly obvious that given an action description, the construction of an equivalent DEL event model is neither straightforward nor systematic. This, we believe, is an argument in favour of our framework, in which many actions can be defined very succinctly and in a natural manner.

\subsubsection{Discussion: DEL vs. EL-O}

The comparison with DEL leads us to a fundamental question: how should an action be described? All the approaches in the literature advo- 


\footnotetext{
${ }^{9}$ In DEL-based planning this distinction is clarified for the first time in [65].

${ }^{10}$ There might be more, such as that the wires leading to the light bulb are working, that the light bulb is not broken etc.: we here neglect the qualification problem [66] which is that the precondition is typically an infinite conjunction.
} 
seen as an epistemic version of the ramification problem [66].

Contrasting with DEL event models, our EL-O-based action descriptions do not require to fully describe all possible ramifications: the direct effect of pushing the light button is simply that the light is on, and the indirect epistemic effects follow from the description of the state, namely who observes the light bulb and who doesn't. This is a fundamentally different answer to the question of how actions should be described. It makes the modeller's task much simpler and more natural. (A similar argument was first put forward in [67].)

To sum it up, we believe that our action descriptions are a more natural way of modelling action types... at least when this can be done. For example, the public announcement of the disjunction $p \vee q$ (the event model PubAnn $(p \vee q)$ ) or the assignment of $q$ to $p$ (the event model Assign $(p, q)$ ) cannot be captured. This is because all propositional variables are independent in our approach, as witnessed by the fact that the knowledge operator distributes over disjunctions of literals: for different $p$ and $q, K_{i}(p \vee q)$ is equivalent to $K_{i} p \vee K_{i} q$, and $K_{i}(p \leftrightarrow q)$ is equivalent to $\left(K_{i} p \wedge K_{i} q\right) \vee\left(K_{i} \neg p \wedge K_{i} \neg q\right)$. This is clearly a limitation of our approach. In particular, we cannot capture the muddy children problem, in which the children learn that one of them has a muddy forehead without knowing who. Note that while DEL is more general as far as preconditions are concerned (any complex formula can be announced), its postconditions are restricted: they assign propositional variables and therefore cannot model actions with disjunctive ontic effects such as $p \vee q$.

For the same reason we cannot capture the poisonous liquid example from [45]. In this problem there is a bottle of liquid, a healthy lawn, and two actions: pour and senseLawn. The first is the action of pouring some liquid on the lawn that we have already described in Example 8 (Section 5.3). The second senses whether or not the lawn is dead: we have pre(senseLawn) $=T$ and eff(senseLawn) $=\left\{\left\langle T,\left\{S_{i}\right.\right.\right.$ Dead $\left.\left.\}, \emptyset\right\rangle\right\}$. Then for the set of actions Act $=$ \{pour, senseLawn\}, there is no solution of the planning task 


$$
\left\langle\mathrm{OA}, \text { Act },\left\{\neg \text { Dead, } S_{i} \text { Dead }\right\}, S_{i} \text { Poisonous }\right\rangle \text {. }
$$

In particular, pouring some liquid on the lawn and checking whether the lawn is dead does not inform the agent whether Poisonous is the case. This is because after pouring the liquid the agent should know that the state of the lawn is tied to the toxicity of the liquid. Such knowledge cannot be captured in our framework.

An interesting avenue for future research are implicitly coordinated plans as introduce by Bolander et al. [59]. For example, suppose agent 2 would like to borrow the apartment of his friend 1 while 1 is away on vacation. Agent 1 has an action putMat of putting the key under the door mat that is described by pre(putMat) $=T$ and eff (putMat) $=\{\langle T,\{$ Mat $\}, \emptyset\rangle\}$; agent 2 has an action tryTake of trying to take the key with pre(tryTake) $=T$ and eff $($ tryTake $)=\left\{\left\langle T,\left\{S_{2}\right.\right.\right.$ Mat $\left.\}, \emptyset\right\rangle,\left\langle\right.$ Mat, $\left\{\right.$ hasKey $\left.\left.\left._{2}\right\}, \emptyset\right\rangle\right\}$. The initial state is $s_{0}=\emptyset$.

1175 Then 1 putting the key under the mat and 2 taking the key solves the planning task

$$
\left\langle\mathrm{OA}, A c t, s_{0} \text {, hasKey }{ }_{2}\right\rangle .
$$

However, when 2 arrives at the apartment he will not know that the key is under the mat, unless 1 has told him. A better plan, baptised 'implicitly coordinated' in [59], involves 1's action infoMat which can be described in EL-O terms by pre(infoMat $)=T$ and eff $($ try Take $)=\left\{\left\langle T,\left\{S_{2}\right.\right.\right.$ Mat $\left.\left.\}, \emptyset\right\rangle\right\}$. The formal characterisation of such plans requires a language with action operators in which one can describe solutions in the language as sequences of actions, prefixing each action of agent $i$ by $K_{i}$ : it is not enough that the action leads to the goal, the acting agent must also know that. This fails to be the case for 2's action tryTake: as 2 does not know whether the key is under the mat, he does not know whether the conditional effect $\left\langle\right.$ Mat, $\left\{\right.$ hasKey $\left.\left._{2}\right\}, \emptyset\right\rangle$ will be triggered. The extension of EL-O with dynamic modal operators, DEL-PAO, has such operators and should therefore enable reasoning about implicitly coordinated plans. The details however remain to be worked out. 

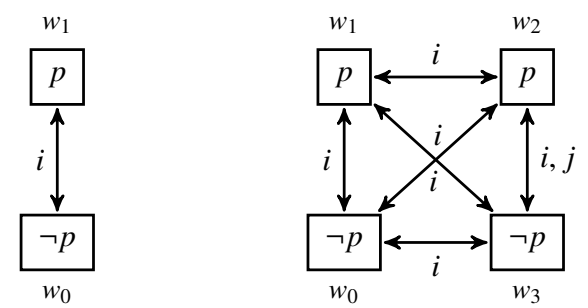

Figure 12: Models $M$ (left) and $M^{\prime}$ (right); reflexive arrows omitted.

\subsection{Muise et al.}

Muise et al. [25] follow a strategy that is very similar to ours where they restrict formulas to boolean combinations of epistemic literals: sequences of belief operators and negations that are followed by a propositional variable; in other terms, formulas of $\mathcal{L}_{\mathrm{EL}}$ without conjunctions and disjunctions. So their grammar of epistemic literals is:

$$
\lambda::=p|\neg \lambda| K_{i} \lambda
$$

where $p$ ranges over $\mathbb{P}$ and $i$ over $\mathbb{A}$. Muise et al.'s proper epistemic knowledge bases (PEKBs) are conjunctions of such epistemic literals [25]. The authors have also started to consider the integration of 'knowing-whether' operators in [68], but have not integrated this into the planning formalism of [25].

The major advantage of our EL-O-based approach over the epistemic literals-based approach is that boolean combinations of epistemic literals cannot express $K_{i}\left(K_{j} p \vee K_{j} \neg p\right) \wedge \neg K_{i} p \wedge \neg K_{i} \neg p$ is true, that is, $i$ knows that $j$ knows whether $p$ while $i$ does not know about $p$ herself. As we had argued in Section 2, such situations are important in interaction and more specifically in communication. They can be expressed in our framework by $\mathcal{L}_{\text {bool }}(\mathrm{OA})$ formulas of the form $S_{i} S_{j} p \wedge \neg S_{i} p$. To prove this formally it suffices to show that $K_{i}\left(K_{j} p \vee K_{j} \neg p\right)$ cannot be expressed by boolean combinations of epistemic literals. 
Proposition 16. Let $i$ and $j$ be different. Then there is no boolean combination of epistemic literals $\varphi$ that is equivalent to $K_{i}\left(K_{j} p \vee K_{j} \neg p\right)$ in S5 Kripke models.

Proof. Consider the two S5 Kripke models $M=\left\langle W, \sim_{\mathbb{A}},\left\{\sim_{i}\right\}_{i \in \mathbb{A}}, V\right\rangle$ and $M^{\prime}=$ $\left\langle W^{\prime}, \sim_{\mathbb{A}}^{\prime},\left\{\sim_{i}^{\prime}\right\}_{i \in \mathbb{A}}, V^{\prime}\right\rangle$ with

$$
\begin{aligned}
W & =\left\{w_{0}, w_{1}\right\}, & W^{\prime} & =\left\{w_{0}, w_{1}, w_{2}, w_{3}\right\}, \\
\sim_{\mathbb{A}} & =W \times W=\sim_{i}, & \sim_{\mathbb{A}}^{\prime} & =W^{\prime} \times W^{\prime}=\sim_{i}^{\prime}, \\
\sim_{j} & =\delta_{W}, & \sim_{j}^{\prime} & =\delta_{W^{\prime}} \cup\left\{\left\langle w_{2}, w_{3}\right\rangle,\left\langle w_{3}, w_{2}\right\rangle\right\}, \\
V\left(w_{0}\right) & =\emptyset \text { and } V\left(w_{1}\right)=\{p\} ; & V^{\prime}\left(w_{0}\right) & =V^{\prime}\left(w_{3}\right)=\emptyset \text { and } V^{\prime}\left(w_{1}\right)=V^{\prime}\left(w_{2}\right)=\{p\} ;
\end{aligned}
$$

where $\delta_{W}=\left\{\left\langle w_{0}, w_{0}\right\rangle,\left\langle w_{1}, w_{1}\right\rangle\right\}$ is the identity relation of $W$ and $\delta_{W^{\prime}}$ is the identity relation of $W^{\prime}$. They are depicted in Figure 12 Clearly, $M, w_{0} \vDash$ $K_{i}\left(K_{j} p \vee K_{j} \neg p\right)$ and $M^{\prime}, w_{0} \not \models K_{i}\left(K_{j} p \vee K_{j} \neg p\right)$. We prove that no boolean combinations of epistemic literals can tell the pointed models $\left(M, w_{0}\right)$ and $\left(M^{\prime}, w_{0}\right)$ apart. From that it immediately follows that there can be no such boolean combination that is equivalent to $K_{i}\left(K_{j} p \vee K_{j} \neg p\right)$.

To establish that of $\left(M, w_{0}\right)$ and $\left(M^{\prime}, w_{0}\right)$ satisfy the same formulas as far as the language of boolean combinations of epistemic literals is concerned it suffices to prove that $M, w_{0} \vDash \lambda$ iff $M^{\prime}, w_{0} \vDash \lambda$ for every epistemic literal $\lambda$. We use induction on the length of $\lambda$. We have to check the following exhaustive list of cases; each of them is straightforward.

${ }_{1220}$ Case 1. $\lambda=q$ for some $q \in \mathbb{P}$. Obvious. ( $M, w_{0} \not \neq \lambda$ and $M^{\prime}, w_{0} \not \neq \lambda$, regardless whether $q$ equals $p$ or not.)

Case 2. $\lambda=\neg \mu$, for some epistemic literal $\mu$. Straightforward application of the induction hypothesis.

Case 3. $\lambda=K_{i} \mu$. We have the following subcases.

1225 Case 3.1. $\lambda=K_{i} q$ for some $q \in \mathbb{P}$. Obvious. $\left(M, w_{0} \not \forall \lambda\right.$ and $M^{\prime}, w_{0} \not \neq \lambda$.)

Case 3.2. $\lambda=K_{i} \neg \mu$, for some epistemic literal $\mu$. We have to dive deeper into the subcases here.

Case 3.2.1. $\lambda=K_{i} \neg q$. Obvious. $\left(M, w_{0} \not \models \lambda\right.$ and $M^{\prime}, w_{0} \not \neq \lambda$.) 
Case 3.2.2. $\lambda=K_{i} \neg \neg \mu$. We use that $\lambda \leftrightarrow K_{i} \mu$ is valid and apply the induction

Case 3.2.3. $\lambda=K_{i} \neg K_{i} \mu$. We use that $K_{i} \neg K_{i} \mu$ and $\neg K_{i} \mu$ are equivalent in S5 and apply the induction hypothesis.

Case 3.2.4. $\lambda=K_{i} \neg K_{j} \mu$. We do yet another case analysis.

Case 3.2.4.1. $\lambda=K_{i} \neg K_{j} q$. Obvious. $\left(M, w_{0} \not \models \lambda\right.$ and $M^{\prime}, w_{0} \not \models \lambda$.)

1235 Case 3.2.4.2. $\lambda=K_{i} \neg K_{j} \neg \mu$. This is the last case analysis we have to do:

Case 3.2.4.2.1. $\lambda=K_{i} \neg K_{j} \neg q$. Obvious. $\left(M, w_{0} \not \forall \lambda\right.$ and $M^{\prime}, w_{0} \not \neq \lambda$.)

Case 3.2.4.2.2. $\lambda=K_{i} \neg K_{j} \neg \neg \mu$. We use that $\lambda \leftrightarrow K_{i} \neg K_{j} \mu$ is valid and apply the induction hypothesis.

Case 3.2.4.2.3. $\lambda=K_{i} \neg K_{j} \neg K_{i} \mu$. We have $M, w_{0} \vDash \lambda \leftrightarrow K_{i} \mu$ and $M^{\prime}, w_{0} \vDash \lambda \leftrightarrow$ $K_{i} \mu$, allowing us to apply the induction hypothesis.

Case 3.2.4.2.4. $\lambda=K_{i} \neg K_{j} \neg K_{j} \mu$. We use that $K_{i} \neg K_{j} \neg K_{j} \mu$ and $K_{i} K_{j} \mu$ are equivalent in $\mathrm{S} 5$ and apply the induction hypothesis.

Case 3.2.4.3. $\lambda=K_{i} \neg K_{j} K_{i} \mu$. We have $M, w_{0} \vDash \lambda \leftrightarrow K_{i} \mu$ and $M^{\prime}, w_{0} \vDash \lambda \leftrightarrow K_{i} \mu$, allowing us to apply the induction hypothesis.

1245 Case 3.2.4.4. $\lambda=K_{i} \neg K_{j} K_{j} \mu$. We use that $K_{i} \neg K_{j} K_{j} \mu$ and $K_{i} \neg K_{j} \mu$ are equivalent in $\mathrm{S} 5$ and apply the induction hypothesis.

Case 3.3. $\lambda=K_{i} K_{i} \mu$. We use that $K_{i} K_{i} \mu$ and $K_{i} \mu$ are equivalent in S5 and apply the induction hypothesis.

Case 3.4. $\lambda=K_{i} K_{j} \mu$. We have $M, w_{0} \vDash \lambda \leftrightarrow K_{i} \mu$ and $M^{\prime}, w_{0} \vDash \lambda \leftrightarrow K_{i} \mu$, 1250 allowing us to apply the induction hypothesis.

Case 4. $\lambda=K_{j} \mu$. We have $M, w_{0} \vDash \lambda \leftrightarrow \mu$ and $M^{\prime}, w_{0} \vDash \lambda \leftrightarrow \mu$, allowing us to apply the induction hypothesis.

\subsection{Kominis and Geffner}

Kominis and Geffner's approach [53, 69] distinguishes three kinds of actions: physical actions modifying the world, public updates (that are nothing but DEL-like public announcements), and sensing actions by means of which an agent learns whether a formula is true or not. 
On the one hand, their approach is more general than ours because agents can sense arbitrary formulas. This allows them to model the muddy children problem, which we cannot express as a simple epistemic planning task (see Section 8.1.5). However, if we restrict the formulas describing the set of initially possible states, the formulas that are publicly announced, and the formulas that are sensed to observability atoms (more precisely: to the equivalent $\mathcal{L}_{\mathrm{EL}}$ formulas) then everything that can be modelled in Kominis and Geffner's approach can also be in ours.

On the other hand, Kominis and Geffner's approach imposes three severe restrictions:

- all actions can be split up in one of the three categories;

- the set of initially possible states is common knowledge among all agents;

- all physical actions and all public updates are public;

- all sensing actions are semi-private.

The first hypothesis is clearly too strong for many natural everyday situations, as we have already said in Section 4.2 (Remark 5). The second hypothesis is also very strong: it forbids the modelling of private actions modifying the world as required in several of our examples of Section 6 . The third hypothesis means that when an agent $i$ senses the truth value of $\varphi$ then all other agents see this: they learn that $i$ knows whether $\varphi$ is true or not but ignore whether what $i$ has learned is $\varphi$ or $\neg \varphi$. This means that there can be no private communication; in particular, one cannot model what we take to be a paradigmatic epistemic planning task, viz. the gossip problem.

\subsection{Petrick and Bacchus}

There is also an older approach due to Petrick and Bacchus about planning under incomplete information for a single agent [70, 45] which bears some similarities to ours. Their language is first-order but does not have 
epistemic operators. Instead, there are several kinds of knowledge bases by means of which the epistemic status of pieces of information is represented. In particular, there is a 'knowing-that' database whose elements are literals and a 'knowing-whether' database whose elements are atoms. The point of view is subjective, while ours is objective, i.e., the agent's knowledge is represented, but not what is true in the world. Therefore the identity of $K_{i} p$ and $p \wedge K i f_{i} p$ cannot be expressed in their language. Just as the original proposal in [70], our approach does not account for postdiction (deducing the past from the present), as illustrated by Example 8. This is because in our approach the knowledge operator distributes over disjunctions of literals. This limitation of our approach is also the reason why we cannot account for the muddy children problem: the latter requires actions where the children learn a disjunction, namely that one of them is dirty. A solution to the muddy children problem where public announcements were integrated into DEL-PAO was presented in [7]; however, in that paper only the semantics was designed, without an axiomatisation or complexity result. The study of such mathematical properties will be subject of future work.

\section{Conclusion}

We have made a first step towards realistic multi-agent epistemic planning. Our approach is based on observability atoms, which, we argue, are more appropriate than epistemic literals as previously proposed in the literature. We have also argued that observability atoms provide a simple, finite way to represent situations of maximal ignorance, namely by states that equal the empty set, while such situations require infinite Kripke models in standard epistemic logic (cf. Remark 5 in Section 4.2 and Remark 6 in Section 6.1).

We have shown that goals and conditions of our action descriptions can be reduced to boolean formulas, which has allowed us to encode epistemic planning into classical planning. As our reduction is polynomial, we main- 
tain the PSPACE complexity of classical planning. Building on this we have encoded epistemic action descriptions into PDDL and performed some experiments with a PDDL planner in order to find plans efficiently. Moreover, our use of a logic of knowledge together with a state of the art automated planner-which we assume to be correct in the case of classical planning with conditional effects-provides a method for producing plans that are guaranteed to be correct. We have illustrated our approach with several examples, most importantly the generalised gossip problem, which, we argue, is an interesting candidate for a paradigmatic epistemic planning task.

Alternatively we could encode epistemic planning tasks into AnswerSet Programming (ASP). An encoding of several variants of the generalised gossip problem together with an experimental evaluation that fares better than our encoding can be found in [71].

An extension of our approach to parallel planning was recently proposed in [49], based on the account of non-epistemic parallel planning of [72].

The decidability of EL-O-based epistemic planning contrasts with the undecidability of DEL-based epistemic planning, which is the case even for simple fragments. For example, if actions also change the world (and not only the agents' knowledge), then plan existence is undecidable whenever epistemic operators are allowed in preconditions; if actions are purely epistemic, then it is undecidable whenever two agents are involved or the epistemic depth exceeds 2 [16]. We have nevertheless shown that several simple kinds of event models can be captured by our action descriptions (Section 8.1). Moreover and as we have explained there, there is a fundamental difference between the modelling of actions by means of DEL event models on the one hand and by means of action descriptions on the other: in an EL-O-based modelling, the agents' observational abilities are part of the state, while they are part of the action description in a DEL-based modelling. We have argued that our approach is more natural and that DELbased approaches face an epistemic version of the ramification problem.

As we have said in Remark 2, it is not obvious how EL-O's operator of 
joint observability of the grand coalition $\mathbb{A}$ should be generalised to joint observability of arbitrary groups. We would like to address this issue in future work.

In this paper we have assumed a centralised approach in which a single

planner decides the actions of all agents. Other approaches have studied the classical gossip problem from a completely different perspective, assuming that all agents are autonomous [5, 73, 74]. An interesting research avenue is to study generalised gossiping as a distributed planning task.

\section{Acknowledgements}

We would like to thank the reviewers who did an excellent job in spotting problematic points and suggesting improvements. Thanks are also due to Julien Vianey who ran the experiments on which we report in Section 7 .

This research was partially funded by TAILOR, a project funded by EU Horizon 2020 research and innovation programme under GA No 952215, as well as by ANITI, funded by the French program "Investing for the Future - PIA3” under Grant agreement no ANR-19-PI3A-0004.

\section{References}

[1] S. Khuller, Y. A. Kim, Y. J. Wan, On generalized gossiping and broadcasting (extended abstract), in: G. Di Battista, U. Zwick (Eds.), Algorithms - ESA 2003, 11th Annual European Symposium, Budapest, Hungary, September 16-19, 2003, Proceedings, Vol. 2832 of Lecture Notes in Computer Science, Springer, 2003, pp. 373-384.

[2] D. Liben-Nowell, Gossip is synteny: Incomplete gossip and the syntenic distance between genomes, J. Algorithms 43 (2) (2002) 264-283.

[3] S. M. Hedetniemi, S. T. Hedetniemi, A. L. Liestman, A survey of gossiping and broadcasting in communication networks, Networks 18 (4) (1988) 319-349. doi:10.1002/net.3230180406. 
[4] M. C. Cooper, A. Herzig, F. Maffre, F. Maris, P. Régnier, The epistemic gossip problem, Discrete Mathematics 342 (3) (2019) 654-663. URL https://doi.org/10.1016/j.disc.2018.10.041

[5] M. Attamah, H. van Ditmarsch, D. Grossi, W. van der Hoek, Knowledge and gossip, in: Proceedings of the 21st European Conference on Artificial Intelligence (ECAI 2014), 2014, pp. 21-26.

[6] A. Herzig, E. Lorini, F. Maffre, A poor man's epistemic logic based on propositional assignment and higher-order observation, in: W. van der Hoek, W. H. Holliday, W.-f. Wang (Eds.), Proceedings of the 5th International Conference on Logic, Rationality and Interaction (LORI 2015), Springer Verlag, 2015, pp. 156-168.

URL http://www.irit.fr/ Andreas.Herzig/P/Lori15.html

[7] T. Charrier, A. Herzig, E. Lorini, F. Maffre, F. Schwarzentruber, Building epistemic logic from observations and public announcements, in: C. Baral, J. P. Delgrande, F. Wolter (Eds.), Principles of Knowledge Representation and Reasoning: Proceedings of the Fifteenth International Conference, KR 2016, Cape Town, South Africa, April 25-29, 2016, AAAI Press, 2016, pp. 268-277.

URL http://www.aaai.org/ocs/index.php/KR/KR16/paper/ view/12899

[8] A. Herzig, E. Lorini, F. Maffre, F. Schwarzentruber, Epistemic boolean games based on a logic of visibility and control, in: S. Kambhampati (Ed.), Proceedings of the Twenty-Fifth International Joint Conference on Artificial Intelligence, IJCAI 2016, New York, NY, USA, 9-15 July 2016, IJCAI/AAAI Press, 2016, pp. 1116-1122.

URL http: / / www.ijcai.org/Abstract/16/162

口[9] F. Maffre, Ignorance is bliss: observability-based dynamic epistemic logics and their applications. (le bonheur est dans l'ignorance : logiques épistémiques dynamiques basées sur l'observabilité et leurs 
applications), Ph.D. thesis, Paul Sabatier University, Toulouse, France (2016).

URL https://tel.archives-ouvertes.fr/tel-01488408

[16] G. Aucher, T. Bolander, Undecidability in epistemic planning, in:

[17] T. Bolander, M. H. Jensen, F. Schwarzentruber, Complexity results in epistemic planning, in: Q. Yang, M. Wooldridge (Eds.), Proceedings of 
the Twenty-Fourth International Joint Conference on Artificial Intelligence, IJCAI 2015, Buenos Aires, Argentina, July 25-31, 2015, AAAI Press, 2015, pp. 2791-2797.

URL http://ijcai.org/Abstract/15/395

[18] S. L. Cong, S. Pinchinat, F. Schwarzentruber, Small undecidable problems in epistemic planning, in: J. Lang (Ed.), Proceedings of the Twenty-Seventh International Joint Conference on Artificial Intelligence, IJCAI 2018, July 13-19, 2018, Stockholm, Sweden, ijcai.org, 2018, pp. $4780-4786$.

URL https://doi.org/10.24963/ijcai.2018/664

[19] T. Bolander, T. Charrier, S. Pinchinat, F. Schwarzentruber, Del-based epistemic planning: Decidability and complexity, Artif. Intell. 287 (2020) 103304. doi:10.1016/j.artint.2020.103304. URL https://doi.org/10.1016/j.artint.2020.103304

[20] R. Demolombe, M. del Pilar Pozos Parra, A simple and tractable extension of situation calculus to epistemic logic, in: Z. W. Ras, S. Ohsuga (Eds.), Foundations of Intelligent Systems, 12th International Symposium, ISMIS 2000, Charlotte, NC, USA, October 11-14, 2000, Proceedings, Vol. 1932 of Lecture Notes in Computer Science, Springer, 2000, pp. $515-524$.

URL https://doi.org/10.1007/3-540-39963-1_54

[21] T. C. Son, C. Baral, Formalizing sensing actions A transition function based approach, Artif. Intell. 125 (1-2) (2001) 19-91. URL https://doi.org/10.1016/S0004-3702(00)00080-1

[22] M. Steedman, R. P. Petrick, Planning dialog actions, in: Proceedings of the 8th SIGDIAL Workshop on Discourse and Dialogue (SIGdial 2007), 2007, pp. 265-272.

[23] G. Lakemeyer, Y. Lespérance, Efficient reasoning in multiagent epistemic logics, in: L. D. Raedt, C. Bessière, D. Dubois, P. Doherty, P. Fras- 
coni, F. Heintz, P. J. F. Lucas (Eds.), ECAI 2012 - 20th European Conference on Artificial Intelligence. Including Prestigious Applications of Artificial Intelligence (PAIS-2012) System Demonstrations Track, Montpellier, France, August 27-31 , 2012, Vol. 242 of Frontiers in Artificial Intelligence and Applications, IOS Press, 2012, pp. 498-503.

URL https://doi.org/10.3233/978-1-61499-098-7-498

[24] H. Palacios, H. Geffner, Compiling uncertainty away: Solving conformant planning problems using a classical planner (sometimes), in: Proceedings, The Twenty-First National Conference on Artificial Intelligence and the Eighteenth Innovative Applications of Artificial Intelligence Conference, July 16-20, 2006, Boston, Massachusetts, USA, AAAI Press, 2006, pp. 900-905.

URL http://www.aaai.org/Library/AAAI/2006/aaai06-142. php

[25] C. Muise, V. Belle, P. Felli, S. A. McIlraith, T. Miller, A. R. Pearce, L. Sonenberg, Planning over multi-agent epistemic states: A classical planning approach, in: Proceedings of the 29th AAAI Conference on Artificial Intelligence (AAAI 2015), AAAI Press, 2015, pp. 3327-3334.

[26] J. Fan, Y. Wang, H. van Ditmarsch, Contingency and knowing whether, Rew. Symb. Logic 8 (1) (2015) 75-107. URL https://doi.org/10.1017/S1755020314000343

[27] K. Su, A. Sattar, X. Luo, Model checking temporal logics of knowledge via OBDDs, The Computer Journal 50 (4) (2007) 403-420.

[28] W. van der Hoek, P. Iliev, M. Wooldridge, A logic of revelation and concealment, in: W. van der Hoek, L. Padgham, V. Conitzer, M. Winikoff (Eds.), Proceedings of the 11th International Conference on $\mathrm{Au}-$ tonomous Agents and Multiagent Systems (AAMAS 2012), IFAAMAS, 2012, pp. 1115-1122.

URL http://dl.acm.org/citation.cfm?id=2343856 
[29] J. van Benthem, J. van Eijck, M. Gattinger, K. Su, Symbolic model checking for dynamic epistemic logic - S5 and beyond, J. Log. Comput. 28 (2) (2018) 367-402. URL https://doi.org/10.1093/logcom/exx038

[30] W. Lenzen, Recent work in epistemic logic, North Holland Publishing Company, Amsterdam, 1978.

[31] W. Lenzen, On the semantics and pragmatics of epistemic attitudes, in: A. Laux, H. Wansing (Eds.), Knowledge and belief in philosophy and AI, Akademie Verlag, Berlin, 1995, pp. 181-197.

[32] W. H. Holliday, Epistemic logic and epistemology, in: Introduction to Formal Philosophy, Springer, 2018, pp. 351-369.

[33] M. Cooper, A. Herzig, F. Maffre, F. Maris, E. Perrotin, P. Regnier, When 'knowing whether' is better than 'knowing that', in: 13èmes Journées d'Intelligence Artificielle Fondamentale (JIAF 2019), hal-02302984, 2019.

[34] A. Herzig, E. Perrotin, On the axiomatisation of common knowledge, in: Proceedings of the 13th conference on Advances in Modal Logic (AiML 2012), College Publications, 2020.

[35] P. Blackburn, M. de Rijke, Y. Venema, Modal Logic, Cambridge Tracts in Theoretical Computer Science, Cambridge University Press, 2001.

[36] A. Herzig, F. Maffre, How to share knowledge by gossiping, in: Proceedings of the 3rd International Conference on Agreement Technologies (AT 2015), Springer-Verlag, 2016, pp. 249-263.

[37] M. R. Genesereth, N. J. Nilsson, Logical Foundations of Artificial Intelligence, Morgan Kaufmann Publishers, 1989.

[38] M. Baldoni, L. Giordano, A. Martelli, A modal extension of logic programming: Modularity, beliefs and hypothetical reasoning, J. Log. 
Comput. 8 (5) (1998) 597-635. doi:10.1093/logcom/8.5.597.

[39] M. Baldoni, Normal multimodal logics: Automatic deduction and logic programming extension, Ph.D. thesis, Universit $\tilde{A}$ degli Studi di Torino, Dipartimento di Informatica (1998).

[40] E. Lorini, A. Herzig, Direct and indirect common belief, in: A. Konzelmann Ziv, H. B. Schmid (Eds.), Institutions, Emotions, and Group Agents: Contributions to Social Ontology, Springer, 2014, pp. 355-372.

[41] A. Herzig, Logics of knowledge and action: critical analysis and challenges, Auton. Agents Multi Agent Syst. 29 (5) (2015) 719-753. URL https://doi.org/10.1007/s10458-014-9267-z

[42] S. N. Artemov, Observable models, in: S. N. Artemov, A. Nerode (Eds.), Logical Foundations of Computer Science - International Symposium, LFCS 2020, Deerfield Beach, FL, USA, January 4-7, 2020, Proceedings, Vol. 11972 of Lecture Notes in Computer Science, Springer, 2020, pp. $12-26$. URL https://doi.org/10.1007/978-3-030-36755-8_2

[43] A. Herzig, E. Lorini, F. Maffre, Possible worlds semantics based on observation and communication, in: H. van Ditmarsch, G. Sandu (Eds.), Jaakko Hintikka on Knowledge and Game-Theoretical Semantics, Springer International Publishing, Cham, 2018, pp. 339-362. URL https: / /doi.org/10.1007/978-3-319-62864-6_14

[44] H. Geffner, B. Bonet, A Concise Introduction to Models and Methods for Automated Planning, Synthesis Lectures on Artificial Intelligence and Machine Learning, Morgan \& Claypool Publishers, 2013.

[45] R. P. A. Petrick, F. Bacchus, Extending the knowledge-based approach to planning with incomplete information and sensing, in: D. Dubois, 
C. A. Welty, M. Williams (Eds.), Principles of Knowledge Representation and Reasoning: Proceedings of the Ninth International Conference (KR2004), Whistler, Canada, June 2-5, 2004, AAAI Press, 2004, pp. $613-622$. URL http: / /www .aaai .org/Library/KR/2004/kr04-064.php

[46] B. Baker, R. Shostak, Gossips and telephones, Discrete Mathematics 2 (3) (1972) 191-193. doi:10.1016/0012-365X(72) 90001-5.

[47] R. Tijdeman, On a telephone problem, Nieuw Archief voor Wiskunde 19 (3) (1971) 188-192.

[53] F. Kominis, H. Geffner, Beliefs in multiagent planning: From one agent to many, in: R. I. Brafman, C. Domshlak, P. Haslum, S. Zilberstein (Eds.), Proceedings of the Twenty-Fifth International Conference 
on Automated Planning and Scheduling, ICAPS 2015, Jerusalem,

[54] D. Lewis (Ed.), Convention: A Philosophical Study, Harvard University Press, 1969.

[59] T. Bolander, T. Engesser, R. Mattmüller, B. Nebel, Better eager than

n lazy? How agent types impact the successfulness of implicit coordination, in: Principles of Knowledge Representation and Reasoning: Proceedings of the Sixteenth International Conference, KR 2018,

URL https://aaai.org/ocs/index.php/KR/KR18/paper/ View/18070 
[60] H. van Ditmarsch, A. Herzig, J. Lang, P. Marquis, Introspective forgetting, Synthese 169 (2) (2009) 405-423. URL https://doi .org/10.1007/s11229-009-9554-4

[61] R. Milner, An algebraic definition of simulation between programs, in: D. C. Cooper (Ed.), Proceedings of the 2nd International Joint Conference on Artificial Intelligence. London, UK, September 1-3, 1971, William Kaufmann, 1971, pp. 481-489. URL http: / / jcai.org/Proceedings/71/Papers/044.pdf

[62] T. Bolander, Seeing is believing: Formalising false-belief tasks in dynamic epistemic logic, in: A. Herzig, E. Lorini (Eds.), Proceedings of the European Conference on Social Intelligence (ECSI-2014), Barcelona, Spain, November 3-5, 2014, Vol. 1283 of CEUR Workshop Proceedings, CEUR-WS.org, 2014, pp. 87-107.

URL http://ceur-ws.org/Vol-1283/paper_14.pdf

[63] T. Bolander, Seeing is believing: Formalising false-belief tasks in dynamic epistemic logic, in: H. van Ditmarsch, G. Sandu (Eds.), Jaakko Hintikka on Knowledge and Game-Theoretical Semantics, Springer International Publishing, Cham, 2018, pp. 207-236.

URL https://doi.org/10.1007/978-3-319-62864-6_14

[64] R. Audi (Ed.), The Cambridge Dictionary of Philosophy, Second Edition, Cambridge University Press, 1999, entry 'Action'.

[65] A. O. Liberman, A. Achen, R. K. Rendsvig, Dynamic term-modal logics for first-order epistemic planning, Artif. Intell. 286 (2020) 103305. URL https://doi.org/10.1016/j.artint.2020.103305

[66] J. McCarthy, Epistemological problems of artificial intelligence, in: B. L. Webber, N. J. Nilsson (Eds.), Readings in Artificial Intelligence, Morgan Kaufmann Publishers, 1981, pp. 459 - 465. URL http://www.sciencedirect.com/science/article/pii/ B9780934613033500350 
[67] T. Bolander, H. van Ditmarsch, A. Herzig, E. Lorini, P. Pardo, F. Schwarzentruber, Announcements to attentive agents, Journal of Logic, Language and Information 25 (1) (2016) 1-35. URL https://doi.org/10.1007/s10849-015-9234-3

[68] T. Miller, P. Felli, C. J. Muise, A. R. Pearce, L. Sonenberg, 'Knowing whether' in proper epistemic knowledge bases, in: D. Schuurmans, M. P. Wellman (Eds.), Proceedings of the Thirtieth AAAI Conference on Artificial Intelligence, February 12-17, 2016, Phoenix, Arizona, USA, AAAI Press, 2016, pp. 1044-1050.

a URL http://www.aaai.org/ocs/index.php/AAAI/AAAI16/ paper/view/12291

[69] F. Kominis, H. Geffner, Multiagent online planning with nested beliefs and dialogue, in: L. Barbulescu, J. Frank, Mausam, S. F. Smith (Eds.), Proceedings of the Twenty-Seventh International Conference on Automated Planning and Scheduling, ICAPS 2017, Pittsburgh, Pennsylvania, USA, June 18-23, 2017, AAAI Press, 2017, pp. 186-194.

a URL https://aaai.org/ocs/index.php/ICAPS/ICAPS17/ paper/view/15748

${ }_{1645}$ [70] R. P. A. Petrick, F. Bacchus, A knowledge-based approach to planning with incomplete information and sensing, in: M. Ghallab, J. Hertzberg, P. Traverso (Eds.), Proceedings of the Sixth International Conference on Artificial Intelligence Planning Systems, April 23-27, 2002, Toulouse, France, AAAI, 2002, pp. 212-222.

[71] E. Erdem, A. Herzig, Solving gossip problems using answer set programming: An epistemic planning approach, Electronic Proceedings in Theoretical Computer Science 325 (2020) 52-58, F. Ricca, A. Russo et al. (Eds.): Proc. 36th International Conference on Logic Program- 
ming (Technical Communications) (ICLP 2020), EPTCS 325. doi: $10.4204 /$ eptcs.325.11.

URL http://dx.doi.org/10.4204/EPTCS.325.11

[72] A. Herzig, F. Maris, J. Vianey, Dynamic logic of parallel propositional assignments and its applications to planning, in: S. Kraus (Ed.), Proceedings of the Twenty-Eighth International Joint Conference on Artificial Intelligence, IJCAI 2019, Macao, China, August 10-16, 2019, ijcai.org, 2019, pp. 5576-5582.

URL https://doi.org/10.24963/ijcai.2019/774

[73] H. v. Ditmarsch, D. Grossi, A. Herzig, W. v. d. Hoek, L. B. Kuijer, Parameters for epistemic gossip problems, in: Proc. LOFT 2016, 2016.

[74] H. van Ditmarsch, J. van Eijck, P. Pardo, R. Ramezanian, F. Schwarzentruber, Epistemic protocols for dynamic gossip, J. Applied Logic 20 (2017) 1-31. 